\title{
Expression of Chemosensory Protein (CSP) Structures in Pediculus humanis corporis and Acinetobacter baumannii
}

\author{
Guoxia Liu' ${ }^{1}$, Shousong Yue ${ }^{1}$, Balaji Rajashekar ${ }^{2}$ and Jean-François Picimbon ${ }^{1,3^{*}}$ \\ ${ }^{1}$ Biotechnology Research Center, Shandong Academy of Agricultural Sciences, Jinan, Shandong, China \\ ${ }^{2}$ Institute of Computer Science, Tartu University, Tartu, Estonia
}

${ }^{3}$ School of Bioengineering, QILU University of Technology, Jinan, Shandong, China

Received: 29 April, 2018; Accepted: 8 May, 2019 ; Published: 17 May, 2019

*Corresponding author: Jean-François Picimbon, Biotechnology Research Center, Shandong Academy of Agricultural Sciences, Distinguished Professor, QLUT, Daxue road, Changqing district, Jinan City, Shandong Province, 250363, PR China, Tel: 00-86-531-89631190; Fax: 00-86-53189631776; E-mail: jfpicimbon@163.com \& jfpicimbon@gmail.com

\begin{abstract}
We parsed the microbial genome database using Bombyx mori chemosensory proteins (BmorCSPs) as templates. We extracted eleven bacterial CSPs (B-CSPs) from various microorganisms such as Kitasatospora griseola, K. purpeofusca, K. CB01950, K. MBT66, Escherichia coli, Macrococcus caseolyticus and Acinetobacter baumannii, a known infectious prokaryotic symbiont of various insects, particularly the human body louse. We then parsed the body louse Pediculus humanis corporis genomic database for CSPs. We found six P. humanis corporis (Phum) CSPs all grouped in the same gene cluster. Sequence alignment, structure modeling and phylogenetic analysis of CSP proteins in bacteria and insects reveal duplication, conservation, gene loss, but also diversification and neofunctionalization that took place at a common stage in this ancestral gene family. Phylogenetic analysis of the amino acid sequences also reveals association of CSPs with other prokaryotic gene families, mainly enzymes and secondary metabolites transporters. Their ability to bind lipids and their proved existence and diversity in infectious bacterial prokaryote systems strongly argue for some important general functions in the cellular metabolism process.
\end{abstract}

Keywords: Bombyx; Acinetobacter; Escherichia; Kitasatospora; Macrococcus; human body louse; binding protein.

\section{Introduction}

Interactions of all various organisms with environment largely drive the process of evolution and/or co-evolution for adaptation [1]. "Life originates in simple forms and develops with time into more and more complex systems. Species from reptiles to birds, plants to insects and mammals to human are not fixed all time but evolve as a result of natural selection. All life is related and has descended from a common ancestor: the birds and the bananas, the fishes and the flowers - all related" (Darwin's theory of evolution) [2]. Different species of living organisms can ultimately be traced to a single common ancestor, the Last Universal Common Ancestor (LUCA), which is probably the most ancient colony of bacteria from which all organisms now living on earth has a common descent. Modern genetic such as high throughput gene sequencing that reveals genome, gene structure and protein expression of many various species gives full-support to Lamarck's and Darwin's theories of evolution: a species is not fixed since creation [3]. The analysis of a particular gene family, namely chemosensory proteins or CSPS, strongly supports the inheritance of the hologenome, the genomes of all an organism's symbiotic microbes mixed with its own genome, which contributes to reject the theory of unchangeability in biological life.

Chemosensory Proteins (CSPs) traditionally refer to small soluble binding proteins that are believed to mediate olfactory recognition at the periphery of sensory receptors, similarly to odor-binding proteins or obps $[4,5,6,7]$. CSPs are made of six (-seven) $\propto$-helical chains of about 110 amino acids, including four cysteines that build two small loops, two adjacent disulfide bridges, in the globular "prism-like" functional structure of the protein $[8,9,10,11]$. The CSP structure is not unchangeable. CSPs are characterized by RNA editing and/or post-translational modifications as reported in the silkworm moth, Bombyx mori $[12,13,14,15,16,17]$. In addition, they are capable of breathing or specific conformational changes, which may represent another key feature of this ancestral primitive multifunctional soluble binding protein family [18].

CSP expressing secretions and tissues are notably pheromone gland secretion, antennal branch, mandibles, salivae, venoms, cephalic capsula, eyes, proboscis, thorax and abdomen, head, epidermis, fat body, gut, wings and legs, among many others $[19,20,21,22,23,24,25,26]$. Such a broad pattern in gene expression over such a wide range of sensory and non-sensory fluids or tissues is in strong agreement with a very general basic function for this gene family.

A role of CSPs in insecticide resistance and xenobiotic degradation has been clearly brought up by Xuan et al. (2015), 
who showed drastic up-regulation of CSP genes in many various tissues over exposure to abamectin insecticide molecule [27]. The role of CSPs and/or other binding proteins in lipid transport, xenobiotic degradation and insecticide resistance has been brought up further by Liu et al. (2016) in whiteflies [28]. While Xuan et al. showed up-regulation of all CSP genes in response to insecticide exposure in a tissue-dependent manner, Liu et al. showed insecticide-mediated up regulation and interaction of the protein with C18-lipid, demonstrating a metabolic role of CSP in insect defense rather than olfactory functions $[27,28]$. The results of CSP protein bound tightly to fatty acid lipid chains are consistent with up-regulation of CSPs in response to microbial/ viral infection as found in flies [29]. In addition, a function of CSPs in lipid transport is consistent with a crucial role not only in general and innate immunity $[27,28,29]$, but also in development, pheromonogenesis and behavioral phase change transition as described in honeybees, moths, whiteflies and solitarious/ gregarious locusts [12,13,14,15,28,30,31,32,33].

Identification of genomic sequences encoding CSPs in the whitefly B. tabaci indicated horizontal transfer of genetic materials through endosymbiotic bacteria between insects and plants $[28,34]$. Here, we report about the finding of CSP expression in bacterial species such as coccobacillus Acinetobacter baumannii, Macrococcus/Staphylococcus caseolyticus, the filamentous actinomycete Kitasatospora griseola, an Actinobacteria genus in the family Streptomycetaceae, and Escherichia coli (E. coli) which are known as common bacteria from the digestive tract, main prokaryotic secondary metabolites, opportunistic multidrug resistant pathogens, high positive cytochrome c oxidase reactions, and symbionts of multiple insect species. The bacterial species A. baumannii, E. coli and M. caseolyticus CSP sequences are twins or identical twins to BmorCSPs (BmorCSP2, BmorCSP4, BmorCSP6 and BmorCSP15), while Kitasatospora CSPs are more distantly related to $B$. mori CSP and orthologous genes in Coleopteran and Hymenopteran species. Because these bacterial pathogens, A. baumannii, E. coli, Kitasataspora and M. caseolyticus, are mediators of infectious diseases in human and can be carried by insects exclusively feeding on human blood, i.e. lice, we also show the genomic repertoire of CSPs in Pediculus humanis corporis (PhumCSPs). The six PhumCSPs build six different orthology groups. PhumCSPs and the CSP family identified in bacteria (B-CSPs) show the same typical folding, a prism with pairs of antiparallel alpha-helices, which certainly represents a protein structure that existed much before the split of the countless types of bacterial strains. In addition, we show protein structure models only differing in alpha-helical motifs in bacterial CSPs, strongly suggesting that the CSP structure had multifunction and/or acquired new function at a far remote time, probably during the early stages of prokaryote evolution (at least 3.8 billion years ago).

\section{Materials and Methods \\ Identification of bacterial CSPs}

Microbial Genomes at the National Center for Biotechnology Information were used to identify CSP counterparts in the bacterial superkingdom. We looked for bacterial counterparts for each B. mori CSP amino acid sequence. B. mori CSPS are known to be expressed throughout many various tissues in the insect body [21,22,23,24,25,26,27]. BmorCSP expression is known to be drastically up-regulated by insecticide exposure [27]. BmorCSP expression is also known to be regulated by specific post-transcriptional and/or post-translational events $[12,13,14,15,16,17]$. The 3D structure of $B$. mori chemosensory protein-1 (BmorCSP1) in solution is known $[9,10]$. We therefore used BmorCSP1-BmorCSP20 protein query sequence to scan the microbial genome database. Eleven clones (hit rate: 23\%$100 \%$ ) were extracted from bacterial species (Table 1). They were identified as CSP clones on the basis of molecular weight and the number of cysteines at conserved position in the primary structure (Table 1 \& Figure 1).

Table 1: Repertoire of CSP clones in bacteria (NCBI Microbial database; Nov $11^{\text {th }} 2018$ ). *: Transmembrane protein (CSP precursor)

\begin{tabular}{|l|c|c|c|c|c|c|}
\hline \multicolumn{1}{|c|}{ Locus } & Nb aa & Nb Cys & MW (kDa) & Organism & Source & Percent identity (BmorCSP) \\
\hline WP_043907137 & 142 & 4 & 14.7 & Streptomycetaceae & Kitasatospora griseola & 23\% (BmorCSP10) \\
\hline WP_071212566 & 120 & 4 & 13.7 & Moraxellaceae & Acinetobacter baumannii & $99 \%$ (BmorCSP2) \\
\hline WP_071222707 & 87 & 4 & 9.7 & Moraxellaceae & Acinetobacter baumannii & $100 \%$ (BmorCSP2) \\
\hline WP_073810176 & 142 & 4 & 15.1 & Streptomycetaceae & Kitasatospora sp. CB01950 & $22 \%$ (BmorCSP10) \\
WP_083646628 & & & & & & \\
\hline WP_078880044 & 142 & 4 & 16.0 & Streptomycetaceae & Kitasatospora purpeofusca & $46 \%$ (BmorCSP14) \\
\hline WP_082558797 & 142 & 4 & 16.0 & Streptomycetaceae & Kitasatospora sp. MBT66 & $46 \%$ (BmorCSP14) \\
\hline WP_089438515 & 131 & 4 & 14.9 & Enterobacteriaceae & Escherichia coli & $100 \%$ (BmorCSP6) \\
\hline WP_096417339* & 491 & 10 & 50.6 & Xanthomonadaceae & Lysobacter capsici & $72 \%$ (BmorCSP5) \\
\hline WP_120787151 & 127 & 4 & 14.5 & Staphylococcaceae & Macrococcus caseolyticus & $72 \%$ (BmorCSP1) \\
\hline WP-120787152 & 131 & 4 & 14.9 & Staphylococcaceae & Macrococcus caseolyticus & $61 \%$ (BmorCSP2) \\
\hline WP_120787167 & 150 & 4 & 17.2 & Staphylococcaceae & Macrococcus caseolyticus & $49 \%$ (BmorCSP4) \\
\hline WP_120787175 & 122 & 4 & 13.9 & Staphylococcaceae & Macrococcus caseolyticus & $72 \%$ (BmorCSP15) \\
\hline
\end{tabular}




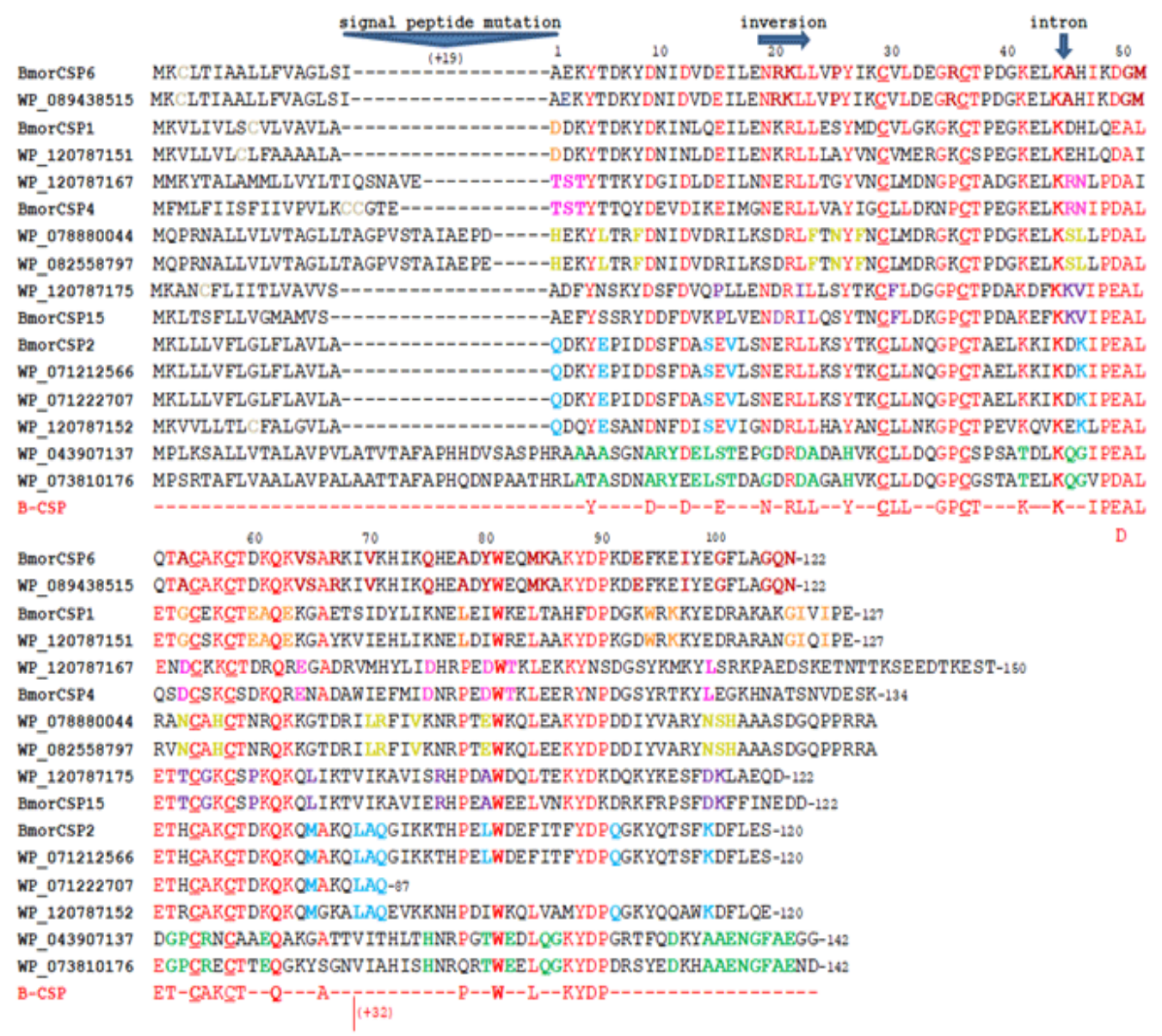

Figure 1: Identification of bacterial CSPs (B-CSPs). The sequences are from Acinetobacter baumannii (WP_071212566/WP_071222707), Escherichia coli (WP_089438515), Kitasatospora griseola (WP_043907137), K. purpeofusca (WP_078880044), K. sp. CB01950 (WP_073810176), K. sp. MBT66 (WP_082558797) and Macrococcus caseolyticus (WP_120787151, WP_120787152, WP_120787167 and WP_120787175; Table 1). Highly conserved amino acids are shown in red. Strictly conserved amino acid residues are shown in red and bold. The four Cys residues characteristic of CSPs are underlined. +Cys residues in signal peptide are shown in bold. Subtype-specific amino acid residues or motifs are shown by different colors. Brown: BmorCSP6/WP_089438515, orange: BmorCSP1/WP_120787151, pink: BmorCSP4/WP_120787167, kaki: WP_078880044/WP_082558797, purple: BmorCSP15/WP_120787175, blue: BmorCSP2/WP_071212566/WP_071222707/WP_120787152, green: WP_043907137/WP_073810176. The typical primary sequence of B-CSPs is shown below the sequence alignment. BmorCSP1, BmorCSP2, BmorCSP4, BmorCSP6 and BmorCSP15 are CSP sequences from the silkworm moth, B. mori $[9,10,12,13,18,26]$. The arrows in blue indicate signal peptide mutation, inversion in NRKL/NKRL motif and intron insertion site after Lysine at position 44, respectively.

Using BmorCSP1 as a template to screen the bacterial genome database extracted Staphylococcaceae Macrococcus caseolyticus WP_120787151(Evalue:2e-54,72\%identity)andWP_120787152 (Evalue: 2e-27, 42\% identity), and WP_089438515 in Enterobacteriaceae Escherichia coli (Evalue: 2e-27, 42\% identity). Using BmorCSP2 as a template extracted WP_071212566 (Evalue: 1e-81, 99\% identity) and WP_071222707 (Evalue: 8e55, 100\% identity) from Acinetobacter calcoaceticus/baumannii complex (Moraxellaceae). A. baumannii WP_071212566 corresponded to a truncated form of WP_071222707/BmorCSP2. Using BmorCSP2 as a template also extracted WP_043907137 (Evalue: 4e-08, 30\% identity) from Kitasatospora griseola, WP_073810176/WP_083646628/OKJ13717 (Evalue: 2e-09, $32 \%$ identity) from $K$. sp. CB01950, and two new clones from $M$. caseolyticus, WP_120787175 (Evalue: 2e-28, 46\% identity) and
WP_120787167 (Evalue: 1e-20, 36\% identity) in addition of McasWP_120787152 (Evalue: 6e-51, 61\% identity) and E. coli WP_089438515 (Evalue: 2e-20, 38\% identity). Using BmorCSP3 did not show other clones, but using BmorCSP4 extracted two additional CSP clones from bacterial strains, Streptomyceteae Kitasatospora sp. MBT66 WP_082558797 (Evalue: 1e-26, 40\% identity) and WP_078880044 from K. purpeofusca (Evalue: 4e$26,40 \%$ identity). Using BmorCSP5 (truncated gene) extracted a CSP clone with an unusual size $(50 \mathrm{kDa})$ and an unusual number of cysteines (10) from Xanthomonadaceae Lysobacter capsici (WP_096417339; Evalue: 9e-10, 52\% identity). WP_096417339 is a hypothetical transmembrane protein. It contains a protein structure related to E. coli TolB protein and a portion of CSP protein $(\propto 1-\propto 3)$ terminated by a RIQPGALADN motif. 
Using BmorCSP6 extracted WP_089438515 from E. coli with an E-value score of $2 \mathrm{e}-85$ and $100 \%$ identity match. Using BmorCSP15 extracted WP_120787175 from M. caseolyticus with an E-value score of $2 \mathrm{e}-61$ and $74 \%$ identity match. Using other BmorCSPs, even BmorCSP10, a CSP clone from B. mori with an unusual size and an unsual number of cysteines (6) and prolines (21), did not extract any supplementary sequences possibly corresponding to CSP (four cysteines, MW: about 10-15 kDa). Using BmorCSP17 extracted a sequence of 3409 amino acids (about $363 \mathrm{kDa}$ ) corresponding to a hypothetical precursor protein for large tegument protein UL36 and retinal protein (pfam15449; WP_121154966) from the actinobacterium
Micromonospora pisi. This sequence did not have the structure (Cysteine profile) characteristic of CSPs, but shared some typical motifs (RLLLG-Y and KYEA) with CSPs. Using BmorCSP consensus sequences or consensus sequences from CSP genes of other insect species did not extract any additional CSPs from bacteria in the general microbial protein blastome. We also blasted the sequences obtained from Baumannii, Kitasatospora and Macrococcus for an iterative search in the microbial protein database. Only twelve bacterial CSP amino acid sequences and the odd WP_096417339 sequence (transmembrane protein and/ or CSP precursor) could be extracted so far (Tables $1 \& 2$ ).

Table 2: Identity matrix (mean percent identity values) between every pair of B-CSPs. ${ }^{*}$ Transmembrane protein (CSP precursor)

\begin{tabular}{|c|c|c|c|c|c|c|c|c|c|c|c|c|}
\hline Locus & 07137 & 12566 & 22707 & 10176 & 80044 & 58797 & 38515 & 87152 & 87167 & 87151 & 87175 & 17339* \\
\hline WP_043907137 & & 25 & 26 & 68 & 27 & 27 & 20 & 25 & 22 & 25 & 30 & 25 \\
\hline WP_071222707 & 26 & 100 & & 26 & 31 & 31 & 39 & 64 & 39 & 39 & 48 & 28 \\
\hline WP_073810176 & 68 & 25 & 26 & & 26 & 26 & 19 & 24 & 25 & 28 & 26 & 19 \\
\hline WP_078880044 & 27 & 29 & 31 & 26 & & 98 & 36 & 31 & 37 & 39 & 30 & 42 \\
\hline WP_082558797 & 27 & 29 & 31 & 26 & 98 & & 35 & 31 & 37 & 38 & 31 & 42 \\
\hline WP_089438515 & 20 & 35 & 39 & 19 & 36 & 35 & & 31 & 32 & 42 & 38 & 37 \\
\hline WP-120787152 & 25 & 60 & 64 & 24 & 31 & 31 & 31 & & 27 & 39 & 42 & 27 \\
\hline WP_120787167 & 22 & 33 & 39 & 25 & 37 & 37 & 32 & 27 & & 43 & 32 & 32 \\
\hline WP_120787151 & 25 & 35 & 39 & 28 & 39 & 38 & 42 & 39 & 43 & & 32 & 31 \\
\hline WP_120787175 & 30 & 45 & 48 & 26 & 30 & 31 & 38 & 42 & 32 & 32 & & 39 \\
\hline WP_096417339* & 25 & 28 & 28 & 19 & 42 & 42 & 37 & 27 & 39 & 31 & 32 & \\
\hline
\end{tabular}

\section{Identification of louse CSPs}

Fly Base and Broad Institute database at http://www. broadinstitute.org (Aedes, Culex and Pediculus) were used to identify CSP sequences from Phthiraptera Pediculus humanus corporis (Human body louse) and retrieve genomic sequences by blasting the nucleotide sequence of each gene against the assembly of scaffolds and contigs $[35,36]$. Not only BmorCSPs but also CSP consensus sequences from different insect species such as Acyrthosiphon pisum, Aedes aegypti, Anopheles gambiae, Apis mellifera, Bemisia tabaci, Culex pipiens, Drosophila melanogaster, Harpegnathos saltator/Camponotus floridanus, Nasonia vitripennis and Tribolium castaneum were used as queries to search for Pediculus humanus corporis chemosensory proteins (PhumCSPs). Consensus cobbler sequences (blocks or motifs) were built using block maker at http://bioinformatics.weizmann. ac.il/blocks. Similarly to our search for bacterial CSPs, PhumCSPs were identified on the basis of molecular weight and the number of cysteines at conserved position in the primary structure (Table 3 \& Figure 4). Exon and intron boundaries were determined by plotting each CSP RNA sequence against the scaffold or contig open reading frame in the search launcher nucleotide algorithm as described for BmorCSPs [27]. Access numbers and scaffolds containing the complete repertoire of PhumCSP genomic DNA sequences (six genes) are reported in Table 3. The degree of identity across PhumCSPs is reported in Table 4.

\section{Phylogenetic analysis of bacterial and insect CSPs}

The protein sequences were selected from our present study about PhumCSPs and bacterial CSPs (B-CSPs), which have been identified in Enterobacteriaceae, Moraxellaceae, Staphylococcaceae, Streptomycetaceae and Xanthomonadaceae.

Other CSP protein sequences were selected among those identified in model insect species such as $A$. mellifera (Amel), $B$. mori (Bmor), B. tabaci (Btab), D. melanogaster (Dmel), H. saltator / C. floridanus (EFN), N. vitripennis (NV) and T. castaneum (Tcas), for which gene structure and/or genome organization were known $[7,12,27,28,34,37,38,39,40,41,42,43,44]$. We added arthropod (crustacean) CSP sequences from the brine shrimp Artemia franciscana (AfraCSP; ABY62736, ABY62738) and the water flea zooplankton Daphnia pulex (DpulCSP1, DpulCSP2; ABH88166, ABH88167). We provided protein structure data analysis using software tools for AfraCSP, DpulCSP1 and DpulCSP2 in our study (see below). AfraCSP, DpulCSP1 and DpulCSP2 melted with BtabCSP1, a protein known to transport fatty acid lipids such as linoleic acid [28]. 
Table 3: Repertoire of CSP genes identified by in silico analysis of Pediculus humanis corporis genome and EST database (Broad Institute database http://www.broadinstitute.org (Aedes, Culex and Pediculus), Vector Base (Bioinformatics Resource for Invertebrate Vectors of Human Pathogens, https://www.vectorbase.org)). Genomic sequence is in italics. *: Scaffold number, **: Locus.

\begin{tabular}{|c|c|c|c|c|}
\hline Locus & Genome size (Mb) & Gene size (bp) & Intron size (bp) & Accession number \\
\hline & 110 & & & \\
\hline PHUM594410 & & 716 & 187 (int1)/100 (int2) & $\begin{array}{c}A A Z 001007241^{*}, 1103172108314, \\
D S 235878^{* *}, N W \_002987883, X_{-} \_002432547 \\
\text { XP_002432592, EEB19854, }\end{array}$ \\
\hline PHUM594420 & & 687 & 288 & $\begin{array}{c}\text { AAZ001007241*, } 1103172108314, D S 235878^{* *}, \\
N W_{-} 002987883, \text { XM_002432548, XP_002432593, } \\
\text { EEB19855 }\end{array}$ \\
\hline PHUM594430 & & 667 & 277 & $\begin{array}{c}A A Z 001007241^{*}, 1103172108314, \text { DS235878**, } \\
\text { NW_002987883,XM_002432549, AJ973467, } \\
\text { XP_002432594, EEB19856, CAJ01514 }\end{array}$ \\
\hline PHUM594540 & & 631 & 208 & $\begin{array}{c}\text { AAZ001007242*, } 1103172108314, D S 235878^{* *}, \\
N W_{-} 002987883, \text { XM_002432550, XP_002432595, } \\
\text { EEB19857 }\end{array}$ \\
\hline PHUM594550 & & 473 & 89 & $\begin{array}{c}\text { AAZ001007242*, 1103172108314, } \\
\text { DS235878**, NW_002987883,XM_002432551, } \\
\text { XP_002432596,EEB19858 }\end{array}$ \\
\hline PHUM594660 & & 491 & 143 & $\begin{array}{c}\text { AAZ001007243*, } 1103172108314, D S 235878^{* *}, \\
N W_{-} 002987883, \text { XM_002432552, XP_002432597, } \\
\text { EEB19859 }\end{array}$ \\
\hline
\end{tabular}

Table 4: Identity matrix (mean percent identity values) between every pair of PhumCSPs.

\begin{tabular}{|c|c|c|c|c|c|c|}
\hline Locus & PHUM594410 & PHUM594420 & PHUM594430 & PHUM594540 & PHUM594550 & PHUM594660 \\
\hline PHUM594410 & & 39 & 42 & 39 & 30 & 37 \\
\hline PHUM594420 & 39 & & 48 & 80 & 31 & 37 \\
\hline PHUM594430 & 42 & 48 & & 45 & 34 & 47 \\
\hline PHUM594540 & 39 & 80 & 45 & & 29 & 30 \\
\hline PHUM594550 & 30 & 31 & 34 & 29 & 37 & \\
\hline PHUM594660 & 37 & 33 & 47 & 30 & & 37 \\
\hline
\end{tabular}

The protein sequences were aligned using MUSCLE (www.drive5.com/muscle) [45]. The phylogenetic trees were constructed on the protein sequence alignment using IQ-TREE (version 1.5.6) [46]. The parameters used to construct the phylogenetic tree were ultrafast bootstrap (UF Boot, using the -bb option of 1000 replicates), and a standard substitution model (-st AA -m TEST), and alrt 1000 -NT AUTO was given to generate the tree. Trees generated from IQ-TREE were visualized using Fig Tree software [47]. For better visualization, trees were modified under the "Trees" section, by applying the increasing order nodes, transform branches to proportional and bootstrap support of greater than 50 were displayed on the branches. The branch-support values were obtained from the output ".tree file".

\section{Construction of 3D structure models}

To compare the CSP structure between bacteria and insects, we used modeling implemented in http://swissmodel.expasy. org/, as a preliminary characterization of functional features that bridge the gap between prokaryotic and eukaryotic systems. $\mathrm{X}$-Ray structure of chemosensory protein A6 in solution (1kx9.1.A) [8] was used as template to predict the structure of each CSP identified in Enterobacteriaceae, Moraxellaceae, Staphylococcaceae, Streptomycetaceae and Phthiraptera, respectively.

MbraA6-1kx9.1A (monomer, 1.6 ) modeled a compact structure made of six $\propto$-helices $(\propto 1-\propto 6)$ [8,9,10,11]. Amino acid 1 is the first residue of the mature protein after removing signal peptide as shown by Edman sequencing at the $\mathrm{N}$-terminus in cockroach and moth CSPs $[5,21]$. We used Clustal to produce an alternative alignment of CSP sequences and then used it as a point of comparison to determine amino acid 1 in bacterial and louse CSPs, respectively. The protein without ligand (apoprotein) was used as a model because specific cognate ligands of CSPs in bacteria and lice remain to be found. 


\section{Results and Discussion}

We searched for "chemosensory" proteins (CSPs) in bacteria. We found a group of genes that share important characteristics, four cysteine codons and similar sequences of RNA building blocks (nucleotides), providing instructions for making products (small soluble alpha-helical proteins) that have a similar structure or function than those identified in insects and arthropods.

Using Bombyx mori chemosensory protein (BmorCSP) sequence as a template to screen the protein library of the bacterial genome database (NCBI) extracted twelve mRNA clones that shared $22-46 \%$ up to $72-100 \%$ identity to BmorCSP clones (see Table 1). In summary, in our search for microbial sequences related to Bombyx CSPs, we extracted one clone (WP_089438515) related to BmorCSP6 in the gut bacteria Escherichia coli, and four clones (WP_120787151, WP_120787152, WP_120787167 and WP_120787175) related to BmorCSP1, BmorCSP2, BmorCSP4 and BmorCSP15, respectively, in Macrococcus caseolyticus, another bacterial genus inhabiting the insect gut. Additionally, we found four clones (WP_043907137, WP_073810176, WP_078880044 and WP_082558797) related to BmorCSP14 and BmorCSP10 in colonic Kitasatospora bacteria, and two clones related to BmorCSP2 (WP_071212566 and WP_071222707) in Acinetobacter baumannii, a known infectious agent for human health, vehiculated by many biting insect species such as the human body louse, Pediculus humanus corporis (Table 1 \& Figure 1). Therefore, the csps that we are describing here are members of a vast protein gene family that exists not only in insects and arthropods, but also in multiple species from the prokaryote superkingdom.

Analyzing the mRNA database from the microbes, eleven different sequences significantly related to CSPs were identified and called B-CSPs to emphasize on the fact that these clones are not from insects or arthropods, but have a common bacterial origin (Table 1 \& Figure 1). The B-CSPs share between 22-98\% identity (Table 2). The four CSPs found in C. caseolyticus display about $27-43 \%$ sequence identity with each other, which is an illustrative example for understanding the specific forms of high molecular diversity in CSPs from a prokaryote system (Table 1 \& Figure 1).

B-CSPs are proteins of about 13.9-17.2 kDa, similarly to moth CSPs and other CSP proteins from other insect species [5-30]. The truncated form of WP_071222707 or BmorCSP2 leads to a protein of about $9.7 \mathrm{kDa}$. This could correspond to a functional protein because the protein is truncated after the intron insertion of CSP (Figure 1). Truncated CSP genes are known to be unexpressed in moths [27], but mutations leading to stop codon mutation and truncated mRNAs have been described, not only in bacteria but also in insects and even in human $[12,13,14,15,16,17,48,49]$. More interestingly, B-CSPs can be divided in seven subtypes, which can be distinguishable from each other on the basis of amino acid sequences (Figure 1). All B-CSPs display four Cysteine residues (Cys29, Cys36, Cys55 and Cys58), Glutamine 62 and Tryptophan 81 at key positions that are conserved across the whole CSP gene family (Figure 1) [7]. Similarly to BmorCSPs, amino acid motifs such as N--RLL-Y (26), GPCT (37), I/LPD/EALET (53), CAKCT (59), KQK-A (66) and KYDP (90) are well-conserved between B-CSPs (Figure 1). The main differences between the seven B-CSP subtypes are found in the $\mathrm{N}$ - and $\mathrm{C}$-terminal regions, and in the central region corresponding to the amino acids at position 5470 , after the IPE/DAL motif. Other key replacements are found at position $46,47,75,78$ and 80 which may underlie subtypespecific functions (Figure 1).

B-CSPs were called B-AbauCSP2, (-32aa) B-AbauCSP2, B-KcbCSP1A, B-KgriCSP1A, B-KmbtCSP14, B-KpurCSP14, B-McasCSP1, B-McasCSP2, B-McasCSP4, B-McasCSP15 and B-EcolCSP6 on the basis of their sequence identity to BmorCSP1, BmorCSP2, BmorCSP14, BmorCSP15 and BmorCSP6, respectively (see Figure 1) $[9,10,12,13,14,15,16,17,21,27]$. The homology modeling structures of B-CSPs was built using $M$. brassicae CSP MbraA6 crystal structure (1kx9.1A) [8]. First, we compared the NMR structure of BmorCSP1 and the structure predicted for BmorCSP1 using $1 \mathrm{kx} 9.1 \mathrm{~A}$ as a reference model $[8,9,10]$. For BmorCSP1, the model and NMR structures only differed in the C-terminal region; the protein folding was the same, which validated our approach of using SWISS-MODEL as a very preliminary analysis of B-CSP structures (Figure 2). In this analysis, the two CSP sequences from the arthropod crustacean daphniid water flea Daphnia pulex (ABH88166, ABH88167; DpulCSP1, DpulCSP2) and the CSP from the extremophile brine shrimp Artemia franciscana (AfraCSP) folded in a compact structure made of five alpha $(\propto)$-helices. The C-terminal tail $(\propto 5)$ paired with $\propto 2$, while $\mathrm{N}$-terminal tail $(\propto 1)$ paired with $\propto 4$, leaving $\propto 3$ at the basis of the triangle CSP structure (Figure 2). Lepidopteran moth CSPs such as BmorCSP1 had a rather different structure. Soluble MbraCSPA6 and BmorCSP1 protein structures display a compact structure made of six alpha $(\propto)$ helices connected by small $\propto-\propto$ loops, as found for the structure of the locust CSPsg4 protein [11]. In this typical CSP structure characterized by six $\alpha$-helices, $\alpha 1 / \alpha 4$ and $\alpha 2 / \alpha 5 / \alpha 6$ align to form the walls of the prism, leaving $\propto 3$ at the basis of the triangle CSP structure, similarly to water flea and shrimp CSPs (Figure 2).

Interestingly, in our survey of B-CSP structure models, the same folding was observed for all B-CSPs, suggesting that this folding of six antiparallel $\alpha$-helices into a compact flexible triangle or prism is rather ancient and expressed not only in eukaryotic organisms, but also in prokaryotic cells. However, some differences were found between the various B-CSP structure models. B-McasCSP2 displayed a long free N-terminal tail. The N-terminal $\alpha$-helix ( $\alpha 1)$ was much shorter than in other B-CSPs (Figure 2). B-McasCSP2 had also shortened $\alpha 2$ and $\alpha 5$, which are two parallel helices on the B-CSP structure (Figure 2). Other differences were found in the number of maillons in $\alpha 1$. The third $\alpha$ maillon was removed in $\alpha 1$ in B-KgriCSP1A, not in B-KcbCSP1A (Figure 2). The first maillon was removed in $\alpha 5$ in B-McasCSP4 (Figure 2). The C-terminus expanded in $\alpha 6$ to form a loop in B-KurCSP14 and B-McasCSP1, similarly to BmorCSP1; all other B-CSP models were lacking this C-terminal loop (Figure 2). $(-32$ aa)B-AbauCSP2 built a small prism with $\mathrm{N}$ - and C-terminal tails folded in two antiparallel $\alpha$-helices in the front of $\alpha 2$, leaving 

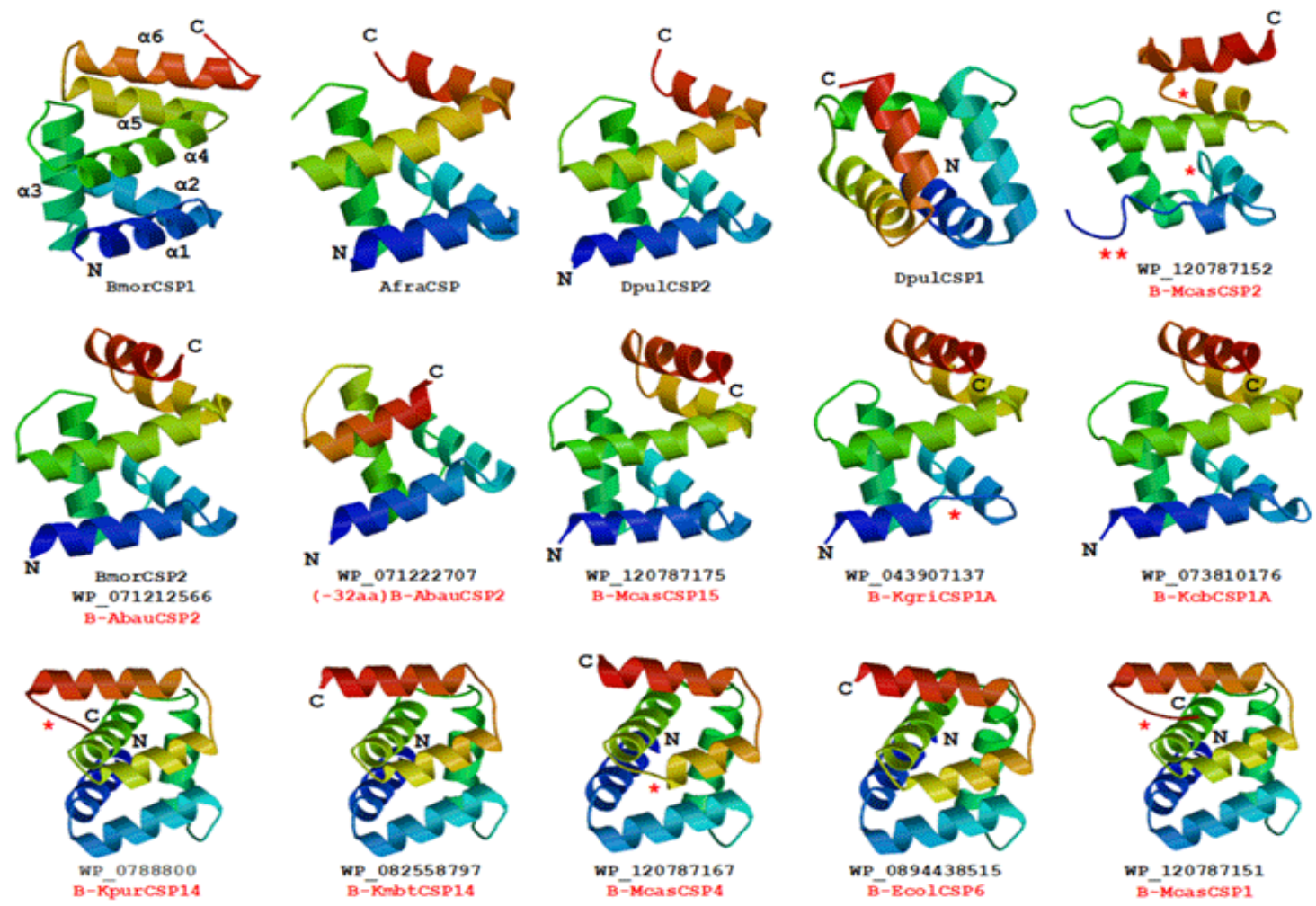

Figure 2: Predicted tertiary structure of B-CSPs. Homology modelling using M. brassicae CSPA6 (1kx9.1.A; X-ray, $1.6 \AA ̊$ ) as template (http://swissmodel.expasy.org) [8]. Afra: Artemia franciscana, Bmor: Bombyx mori, Dpul: Daphnia pulex (Arthropod); Abau: Acinetobacter baumannii, Ecol: Escherichia coli, Kgri: Kitasatospora griseola , Kpur: K. purpeofusca, Kcb: K. sp. CB01950, Kmbt: K. sp. MBT66, Mcas: Macrococcus caseolyticus (Bacteria). The asterisk in red shows mutation sites in protein structure.

$\alpha 3$ at the bottom of the structure like in all other CSPs (Figure 2). These differences in $\alpha$-profiling and C-loop may indicate different functions for B-CSPs.

These results demonstrate the existence of CSP genes not only in Eukaryotes, in arthropods and insects, in winged insects such as moths and in wingless insects such as head and body lice, but also in Prokaryotes, microbial systems, and their expression in many various bacterial strains from E. coli to A. baumannii, therefore across many different types or genera of bacteria, known for severe infectious diseases in human. Interestingly, most of all these bacterial species (Moraxella, Staphylococcus, Streptomyces, Enterobacter and Xanthomonadales) that house CSPs are also known as symbionts of the insect gut $[50,51,52,53,54]$. The gut microbiota plays crucial roles in many various physiological systems such as the growth, development, detoxification, nutrition, immune response and environmental adaptation to insect hosts [55]. Many bacterial species such as E. coli, Staphyloccocus and A. baumannii are known to affect invertebrates and human alike. Furthermore, many insect species are sources of potential human pathogens if not obtained from the environment. This is the case, for instance, of $A$. baumannii, which is known not only as a bacterial pathogen with a long range of various diseases (pneumonia, meningitis, urinary tract and bloodstream infections), but also as a symbiotic species of the head louse and human body louse, Pediculus humanus capitis and Pediculus humanus corporis (humanus) [56,57].

Body louse and head louse are known to infest humans. They are common species of sucking louse in the family Pediculidae (Anoplura, Phthiraptera) that is found where human lives. Like ticks and mites, the head louse is an obligate ectoparasite of humans.

It spends its entire life on the human scalp, not to clean the hairs (cleaning symbiosis) but to feed off the blood from our brain vessels. In addition to bite our scalp from one hair shaft to another hair shaft, they will lay eggs at the bottom of our hairs (called nits). These nits will start a new life cycle and later jump onto new comers. While they feed on us for their health and growth, they will propagate many various epidemic diseases among people, especially young children who will suffer open wounds, scratches, irritations or sores on the head from the constant sucking of blood from the head lice. Head lice infestation usually leads to Pediculosis capitis and Phtiriasis which has been a problem for the human population for thousands years [58].

Besides head louse, there are many other different types of lice that are found on the body, in the armpits or on the pubic area. While they live on different places on the human body, they 
all live on or in the skin of the human body, and they all feed on the human blood and soon can infest the whole body, spreading not only scratches or wounds that will be nests for infectious bacteria, but also more serious pathologies.

Lice are the primary vectors for the transmission of diseases such as epidemic thyphus, trench fever and relapsing fever, which are caused by dangerous strains of bacteria such as Rickettsia prowazekii, Borrelia recurrentis and Bartonella quintana [59]. Therefore, the analysis of potential genes transferred from the bacterium to the louse might be extremely important in the prevention of numerous infectious diseases in human. In particular, it could be that bacteria have passed on CSPs to lice and that lice have passed on CSPs to human through infection of many different sites or tissues.

We checked for the occurrence of horizontal gene transfer of CSPs from bacteria to lice by analyzing the repertoire of CSPs in the Pediculus humanis corporis genome. We found only six CSP genes in the louse genome at six specific loci, namely PHUM594410, PHUM594420, PHUM594430, PHUM594540,
PHUM594550 and PHUM594660 (Figure 3 and Tables $3 \& 4$ ). Such a low number of CSPS was also found in ants, flies, bees, wasps and anopheline mosquitoes ( $\mathrm{n}=4-8)[7,37,38,39,40,41,42,43$, 44]. They are all placed in the same scaffold, which occupies the same chromosomal region of the louse (DS253878; Table 3). Out of the six fully identified Pediculus humanis corporis CSP genes (PhumCSPS), one is found to have two introns (PHUM594410) and the other five genes are found to have a single intron always located at the same position, after Lysine residue at position 44, indicating common ancestry for all these genes (Figure 3). In contrast to BmorCSPs, the intron in PhumCSPs does not vary very much in size. It does not exceed 288 bps (Figure $3 \&$ Table 3). Intron 1 in PHUM594410 is inserted in the signal peptide similarly to AAJJ1196A, BmorCSP19 and GB19453 [27,38,39,43,44]. These 2 introns/ 3 exons genes must be ancestrally related to a CSP gene that occurred in the last common ancestor of moths, beetles, bees and lice, as ancient as more than 300 Mya [60]. Similarly to moth CSPs, PhumCSP genes sit close to each other in the same genomic region separated by about $1500-8500$ bps (Figure 3 ). So the cluster is not a recent expansion of CSPS, but the CSPS have

\section{Pediculus humanus corporis (DS235878)}

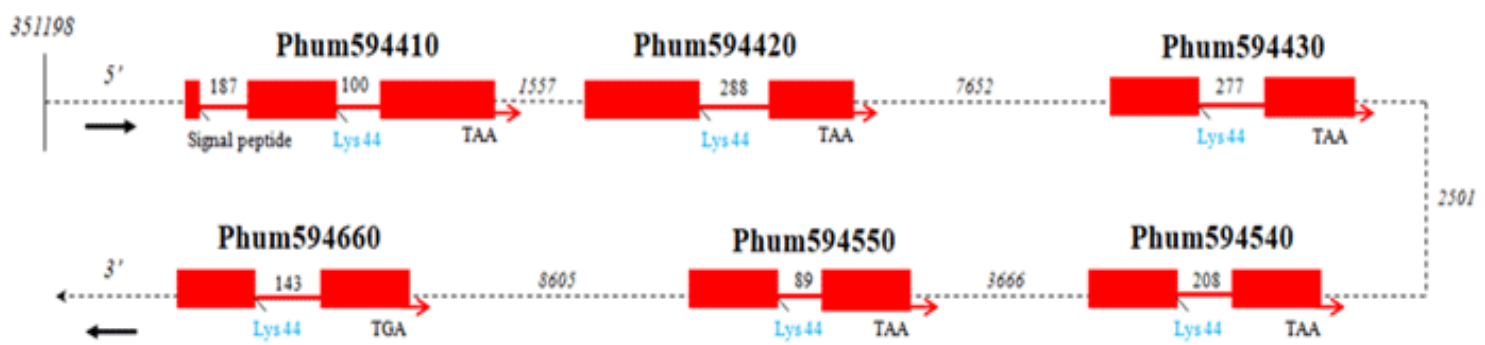

Figure 3: Genomic organization of Pediculus humanis corporis chemosensory protein (PhumCSP) genes on chromosomal region DS253878. PhumCSP genes are shown in red. Exons are shown as red boxes and introns as bold red plain lines. Dotted lines in black are intergenic intron regions. The numbers in italic above the line give the distance between PhumCSPs. The numbers above the intron give the intron size. The amino acid residue in blue shows conserved intron insertion site, after lysine at conserved position (amino acid 44). The arrow indicates the orientation of the gene (5'-3': Phum594410, Phum594420 and Phum594430; 3'-5': Phum594540, Phum594550, Phum594660). Stop codons are indicated, showing that PhumCSPs have either TGA (Phum594660) or TAA stop codons.

remained in this cluster throughout their very long history.

Similarly to Bmor- and B-CSPs, PhumCSPs show about 29$80 \%$ sequence identity with each other (Table 4 \& Figure 4 ). Conservation of specific amino acid residues shows duplication, pairwise evolution, expression and/or function of PhumCSP genes: PHUM594420/PHUM594540, PHUM594410/PHUM594430 and PHUM594550/PHUM594660. However, all PhumCSPs display Threonines T6 and T37, Aspartate 14, Leucine 17, Lysine 44 (intron insertion site), Proline 48, Lysine 77, 21-RLL-23 and 87KYDP-90 motifs, in addition of four Cysteine residues (Cys29, Cys36, Cys55 and Cys58), Glutamine 62 and Tryptophan 81 at key positions that are conserved across the whole CSP gene family [7], including the bacterial CSPs reported in our study (see figures 1 \& 4). The main differences between the PhumCSP subtypes are found in the $\mathrm{N}$ - and C-terminal regions, and in the central region corresponding to the amino acids at position 54-70, after the LPV/E/DAL motif, as found for B-CSPs (Figures $1 \& 4$ ). This corresponds to $\propto$-helix $\propto 4$ on the structure model of PhumCSP (Figure 4).

Like B-CSPs, PHUMCSPs are predicted to fold into a prism characterized by six $\alpha$-helices, with $\alpha 1 / \alpha 4$ and $\alpha 2 / \alpha 5 / \alpha 6$ as square sides, and $\alpha 3$ as a base (Figures $2 \& 4$ ). Interestingly, intron is inserted in $\alpha 3$, and therefore right in the middle of the protein, suggesting that the prism was built by association of two exons, each of them coding for small alpha-helical subunits (Figure 4). This joint seems to have happened much before the time when insect species became diverse and highly specialized, and much before any possible insect-bacteria associations. The degree of identity between insect and bacterial CSPs suggests that 


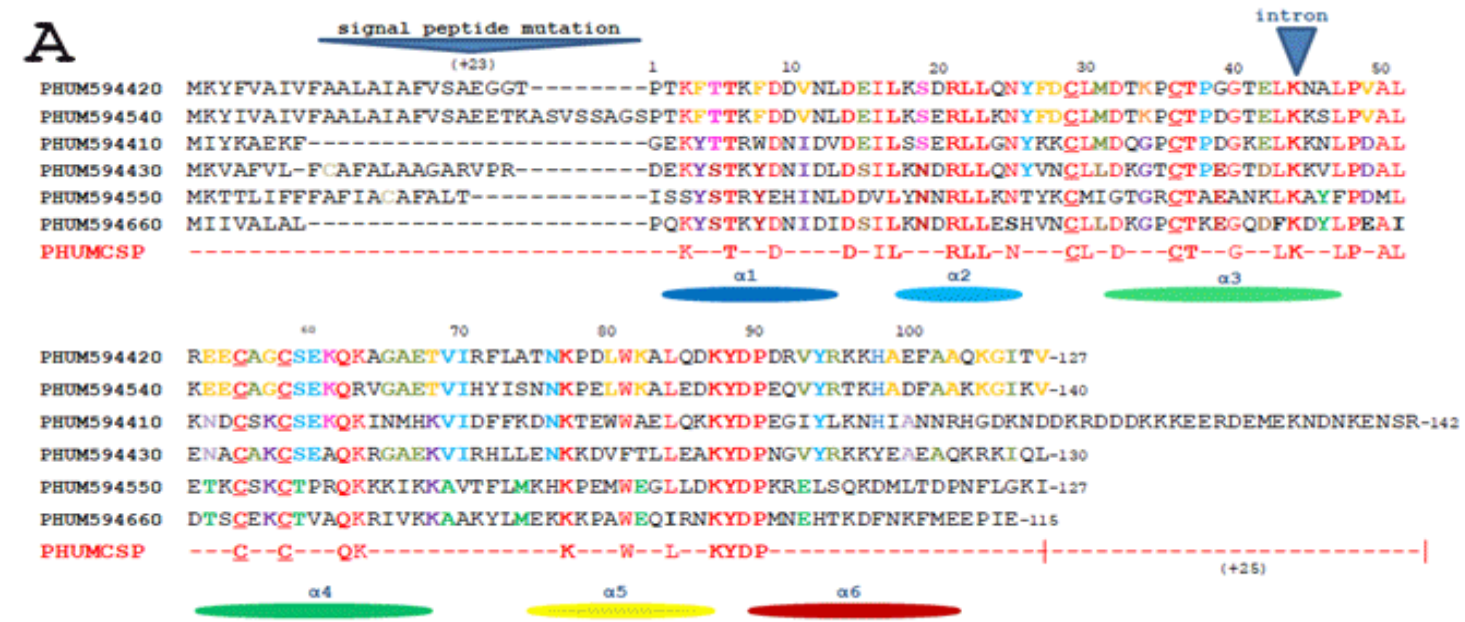

B

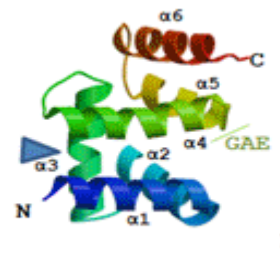

PHUM5 94540

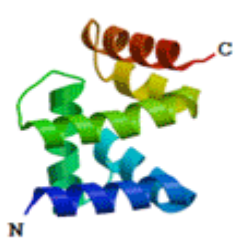

PHUM5 94420

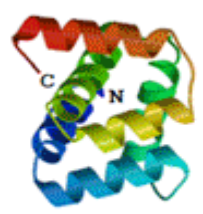

PHUM594430

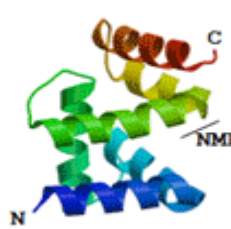

PHUM5 94410

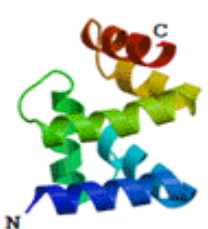

PHUM5 94660

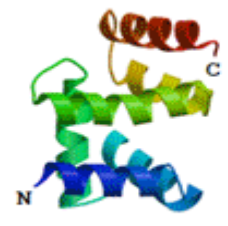

PHUM5 94550

Figure 4: Identification of Pediculus humanis corporis CSPs (PhumCSPs). (A) Sequence alignment of the six PhumCSPs extracted from Pediculus genome: PHUM594410, PHUM594420, PHUM594430, PHUM594540, PHUM594550 and PHUM594660. Sequences are from in silico analysis of Fly Base and Vector Base (Table 3). Highly conserved amino acids are shown in red. Strictly conserved amino acid residues are shown in red and bold. The four Cys residues characteristic of CSPs are underlined. +Cys residues in signal peptide are shown in grey. Subtype-specific amino acid residues or motifs are shown by different colors. Switch to conserved amino acids at position 43, 49, 51 and 84 are shown in black bold. The arrows in blue indicate signal peptide mutation and intron insertion site after Lysine at position 44, respectively. The circles below the alignment show the position of six $\propto$-helices $(\propto 1-\propto 6)$ from model structure. (B) Predicted tertiary structure of PhumCSPs. Homology modelling using MbraCSPA6 (1kx9.1.A; X-ray, 1.6 $\AA$ ) as template (http://swissmodel.expasy.org) [8]. The blue arrow shows the position of intron (inserted in $\propto 3$ at the bottom of the CSP prism). GAE and NMH correspond to subtype-specific motifs (mutation sites) at the extremity of $\propto 4$-helix.

it occurred a long time ago, most likely some Bya. Insect species were even not born or diversified by this time, with sequence evidence reflecting the common structure of CSP, the presence of multiple subtypes and conserved intron insertion sites as early as the rapid emergence and dissemination of various eukaryote and prokaryote cells [61].

We ran a phylogenetic analysis using IQ-TREE to check for the distribution of B-CSPs among PHUMCSPs and CSP sequences from other insect and arthropod species. We found that B-CSPs and PHUMCSPs segregated together at least in two major groups (CSP1 and CSP2), containing also DmelOS-D and BmorCSP15, respectively (Figure 5). PHUM594420 and PHUM594540 were clearly two gene duplicates related to Coleopteran AAJJ0283A [43]. PHUM594420 and PHUM594540 fell closely related to BtabCSP3, a gene from B. tabaci involved in metabolism of toxic plant oil chemicals [28], while PHUM594430 fell closer to AmelASP3c, a gene supposedly involved in pheromone recognition in honeybees [62]. PHUM594550 grouped with hymenopteran CSPs orthologous to AmelGB17875 (Figure 5).
There is no cluster of lice-specific duplications, so PHUMCSPS are not involved in speciation. In addition, the amino acid tree shows that PHUMCSPs might originate from five serial gene duplication events (D1-D5) from PHUM594660 to PHUM594420/ PHUM594540, each of them corresponding to some specific amino acid replacements in the composition of CSP (Figures 3 \& 4). Very importantly, PHUM594660 groups not only with eight BmorCSPs (BmorCSP3, BmorCSP11, BmorCSP12, BmorCSP13, BmorCSP15, BmorCSP17, BmorCSP18 and BmorCSP20) and ant/ wasp CSP gene duplicates, but also with the microbial CSP gene $W P_{-} 120787175$ from the bacterial firmicute bacillus bacillale Staphylococcaceae, M. caseolyticus. This suggests that CSP gene has been duplicated already in the very far common ancestor of bacteria and later on passed on to insects. Also very importantly, bacteria and insects share many other CSP genes such as WP_120787167, WP_078880044, WP_082558797, PHUM594410 and PHUM594420/PHUM594540, which were duplicated or triplicated in lice and beetles for a function that remains to be found. Beetles, moths and bacteria share the same CSP genes such as AAJJ0283B, BmorCSP14, WP_073810176/WP_083646628 


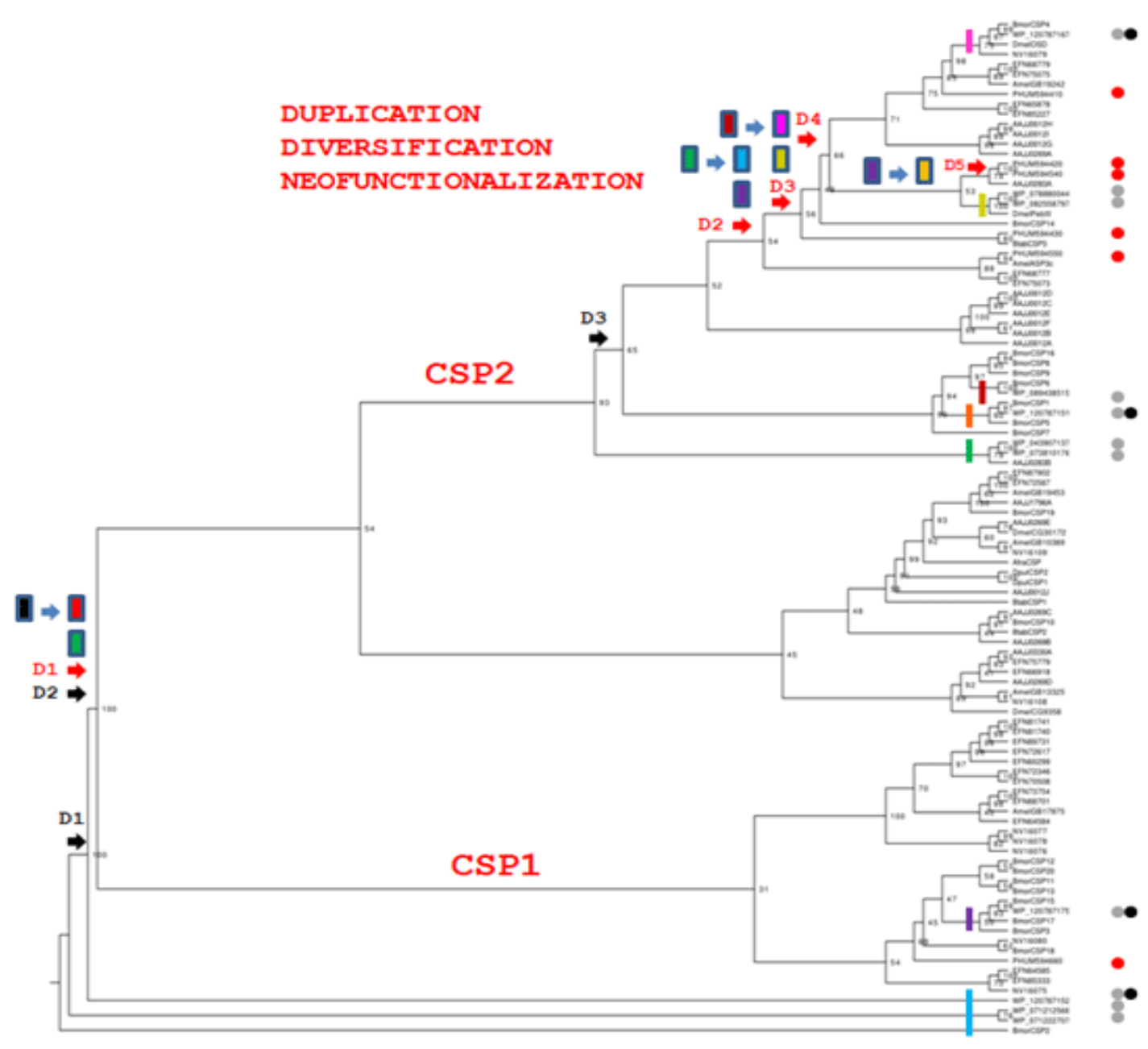

Figure 5: Phylogenetic analysis of bacterial and insect CSP amino acid sequences. Bacterial CSP sequences are those from Figure 1. WP_043: Kitasatospora griseola (streptomycete), WP_071: Acinetobacter baumannii (cocobacillus), WP_073: K. sp. CB01950 (streptomycete), WP_078: K. purpeofusca (streptomycete), WP_082: K. sp. MBT66 (streptomycete), WP_089: Escherichia coli (proteobacter), WP_120: Micrococcus (staphylococcus). PhumCSPs are those from Figure 4. Other insect CSP sequences are from Picimbon [7], Picimbon et al. [21], Honeybee Genome Consortium [38], Forêt et al. [39], Xuan et al. [27], Liu et al. [28, 34,43,44], Bonasio et al. [40], Kulmuni et al. [41] and Zhao et al. [42]. AAJJ: Tribolium castaneum (beetle), Amel: Apis mellifera (bee), Bmor: Bombyx mori (moth), Btab: Bemisia tabaci (whitefly), Dmel: Drosophila melanogaster (fly), EFN: Harpegnathos saltator/ Camponotus floridanus (ant), NV: Nasonia vitripennis (wasp), Phum: Pediculus humanis corporis (louse). Arthropod (crustacean) CSP sequences are from the brine shrimp Artemia franciscana (AfraCSP; ABY62736, ABY62738) and the water flea zooplankton Daphnia pulex: (DpulCSP1, DpulCSP2; ABH88166, ABH88167). Bootstrap values $>50 \%$ after 1000 replicates are shown in support of the branching in IQ-TREE. BmorCSP2 (100\% identity to bacterial counterparts) was used as outgroup. Grey dots indicate the position of B-CSPs. Red dots indicate the position of PhumCSPs. Black dots show the position of the four CSP subtypes identified in Macrococcus (staphylococcus). Black arrows indicate inferred gene-duplication events in bacteria (D1-D3). Red arrows indicate inferred gene-duplication events in insects (D1-D5). Vertical solid lines of different colors indicate different B-CSP subtypes (see Figure 1). Vertical solid squares of different colors indicate amino acid changes for specific motif transition mutation in PhumCSPs (see Figure 4). CSP1 group is a gene copy made at the time of a very early duplication (D1). Transition mutation rate is much higher in the second group of CSPs, CSP2 (D2-D5).

and WP_043907137 (93\% bootstrap). Similarly, bacteria and moths share the same genes WP_089438515 and WP_120787151 (BmorCSP1 and BmorCSP6), which were subsequently subjected to further duplication in the silkworm perhaps as an adaptive response to herbivory and plant chemical detoxification (Figure 5) $[27,28]$.
In CSP1, “Tegument-Retinal protein” WP_121154966 from the bacterial actinobacteria, micromonosporales, Micromonosporaceae, M. pisi grouped with BmorCSP17 (93\% bootstrap; Figure 5). “Transmembrane protein" WP_0964117339 did not group with BmorCSP5 and fell at the bottom of CSP2 group. It may suggest that one group of CSPs diverged from a prominent precursor tegumentretinal protein, while another group of CSPs (CSP2) diverged from a 


\section{Toll-like precursor molecule.}

Most importantly, our phylogenetic analysis suggests that serial duplication events occur not only in insects but also in bacteria. Profiling of gene duplication patterns of pediculus and macrococcus CSPs suggest that the very ancient gene duplication has shaped a CSP1 group that may be important for cell differentiation. The formation of the prokaryotic cell and later the development of multicellular organisms are known to be accompanied by gene expression changes in differentiated cells. Thus, CSP1 may be one of the most ancient form of CSP and may represent a gene set that contributed to the morphological diversity across the bacterial and eukarya superkingdoms and/ or contributed to protect both bacteria and eukaryotes from host plant poisoning $[63,64,65]$. The function of PHUM594660 and WP_120787175 (BmorCSP15) in the CSP protein family has been highly conserved after duplication events and over evolutionary time.

Profiling of gene duplication patterns of pediculus and macrococcus CSPS also suggest that further tandem duplications occurred in both prokaryotes and eukaryotes, leading to diversification and neofunctionalization of the CSP gene family at a common stage of evolution (CSP2; Figure 5). Our phylogenetic analysis of the PHUMCSP family indicates that multiple duplication events have taken place after PHUM594550, at least three (D3, D4 and D5) of which led to the formation of four proteins with novel functions (Figure 5). Comparative analysis of amino acid sequences from PhumCSPs show different steps of sequence mutations. Following duplication 1, CSP acquired specific elements such as N26, L44, V50, L52 and L85. Mutations were also observed in the signal peptide, C-tail and stop codon (Figure 6A). Duplication 2 was accompanied by a few subtle amino acid replacements, but more drastic change occurred in the primary structure of CSP after duplication 3. Over duplication 3, some key residues changed in $\propto 1, \propto 2$ and $\propto 3$, respectively. Signal peptide lost Cys residue and C-terminus acquired a long prominent tail (+25 amino acids) (Figures 4-6A). Thus, CSPs probably acquired diversification and novel function(s) at that time. Interestingly, neofunctionalization and diversification of CSP gene continued over duplication 4 , where mutations were observed mainly in the parallel $\propto$-helices $\propto 1$ and $\propto 4$. C-terminus $(\propto 6)$ changed to A-AA-KGI-V motif (Figures 4-6A). Following duplication 5, the motif corresponding to $\propto 4$ and $\propto 5$ loop was entirely replaced, as found for duplications 3 and 4 (Figures 4-6A). These observations strongly argue for gene duplication and neofunctionalization of the CSP gene family over three steps in the louse genome.

In addition, focusing on PHUMCSPs suggests at least five independent duplication events followed by partial deletions or loss of one copy (Figures 4-6A). Only one CSP gene is present in each orthology group, except for PHUM594540 and PHUM594420 (Figure 5). The topology of the tree and sequence alignment show that the two copies of the genes were preserved at each duplication event, but only one copy of the gene was subsequently subjected to duplication, the other gene remaining with the old function (Figures 4-6). However, the repertoire of CSP sequences expressed in Pediculus cannot be explained by five serial duplications in six genes. Sequence alignment shows conservation of specific residues between PHUM594430 and PHUM594420/PHUM59440 (in green on Figure 4), and a switch from GAE to NMH motif at the extremity of $\propto 4$ in PHUM594410. This can be explained only by a situation where PHUM594430 led to two copies, PHUM594430 with the original GAE motif, and PHUM594410 characterized by GAE substitution by NMH motif and expansion of C-tail (Figure 6B). These two gene copies, PHUM594430 and PHUM594410, were subsequently subjected to duplication. Duplication of PHUM594430 led to PHUM594540 and PHUM594420, while the post-duplication scenario was different for PHUM594410. In the louse genome there is only one copy of PHUM594410 left, and somewhere along evolution, at least Pediculus has lost a duplicated copy of PHUM594410. This gene was not subjected to a further duplication event, so degeneration and gene loss was associated with PHUM594410, while PHUM594430 associated with gene amplification, gene duplication and subsequent modification for neofunctionalization of the CSP gene family (Figure 6B). PhumCSPs fall in the same orthology group than B-CSPs with significantly high bootstrap value (53\%; Figure 5). Therefore, these genetic events that led to CSP diversification might have happened much before the diversification of insect species and the emergence of lice, i.e. much before the split of Eukaryotes and Prokaryote species.

Recent studies indicate possible links between insect CSPs and resistance to biological and chemical toxins [27,28,29]. Outbreaks of louse-borne diseases are usually frequent byproducts of poverty, famine, poor hygiene, poor living conditions, droughts, earthquakes, floats, hurricanes, tornadoes, tsunamis and various other natural disasters, before the advent of insecticides. Largely due to the widespread use of insecticide products such as pyrethroid permethrins for control, a variety of lice has developed resistance to many families of insecticides or pediculicides, resulting in the emergence or re-emergence of lice and lice-diseases in many areas, regions, provinces or states of the world $[66,67,68,69,70]$. The repertoire of (six) CSP genes identified in the body louse $P$. humanis corporis may explain the resistance of the louse to permethrin or pediculicide, following the results from Xuan et al. (2015) and Liu et al. (2016) in moths and whiteflies, respectively, about the role of CSPs in insecticide resistance $[27,28]$. Similarly, the discovery of CSP proteins from $A$. baumannii, M./S. caseolyticus, $K$. griseola and E. coli is important because it may reveal a key mechanism of bactericide resistance in some particular noxious strains mediators of severe infectious diseases [71,72]. Perhaps they went through a mechanism of toxic chemical resistance at an early stage of evolution. CSPs may be involved in the transmission of resistance from one cell to another as found in strains of E. coli that are resistant to one antibiotic and are proved to protect against the same antibiotic the bacterial cells growing nearby [73].

As a first step unraveling the role of CSPs in bacteria, we performed a phylogenetic analysis of B-CSPs not only with insect CSP sequences but also with related genes of various functions. These genes were extracted from microbial database by iterative blast search using CSP as a template. They do not share the 
A

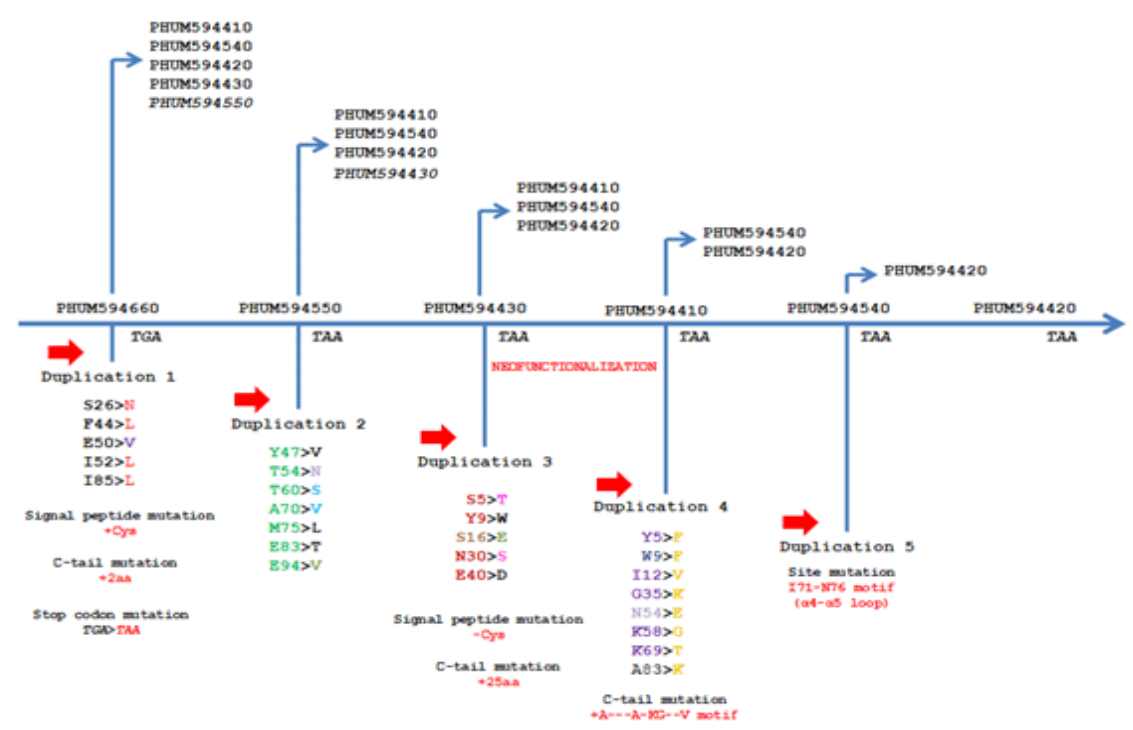

B

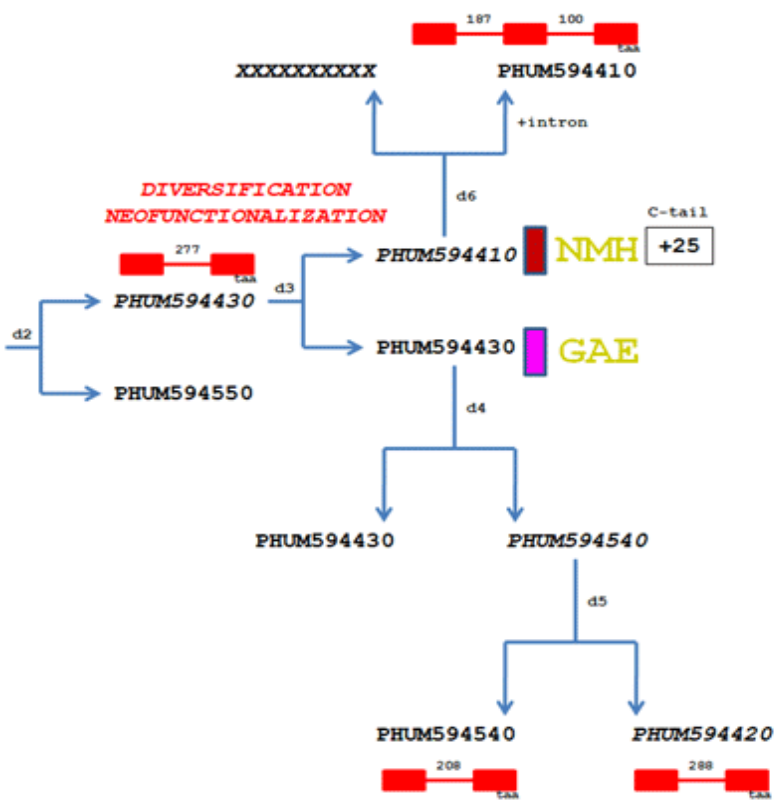

Figure 6: Duplication and transition mutation in PhumCSPs. (A) Point mutations and motif transitions resulting in neofunctionalization in PhumCSP after duplication 3. (B) Inferring evolutionary scenarios in the duplication, loss and diversification of PhumCSP. PHUM594660 corresponds to the original CSP that became a major source of evolutionary innovation because it led to all other PhumCSPs through six gene duplication events (d1-d6). D3 led to the separation of two different subtypes characterized by NMH and GAE, respectively. The GAE-gene copies were preserved and diversified (d3, d4 and d5). The NMH-gene copy was lost following duplication (d6). The NMH-gene was subjected to specific mutation (intron gain), which brought a gene that can be clearly distinguished from the original copy, perhaps in response to major evolutionary transitions in eukaryotic and/or prokaryotic cells.

configuration of disulfide bridges characteristic of CSPs, but they share some amino acid motifs with the CSP gene family [74]. Perhaps most interestingly, the CSPS grouped specifically with genes related to various functions such as DNA-binding proteins, secondary metabolites transporters, pathway signal proteins, phosphatases, amino acid synthases and transcriptional regulators, which may be some examples of protein partners by sub/neofunctionalization of the CSP gene family (Figure 7). The tree is showing a very high bootstrap value for transmembrane protein WP_096417339 and a truncated gene of B. mori, BmorCSP5 (94\%) in a large clade of BmorCSPs together with B-CSPs (51-99\% bootstrap value) (Figure 7). This may suggest that the original 


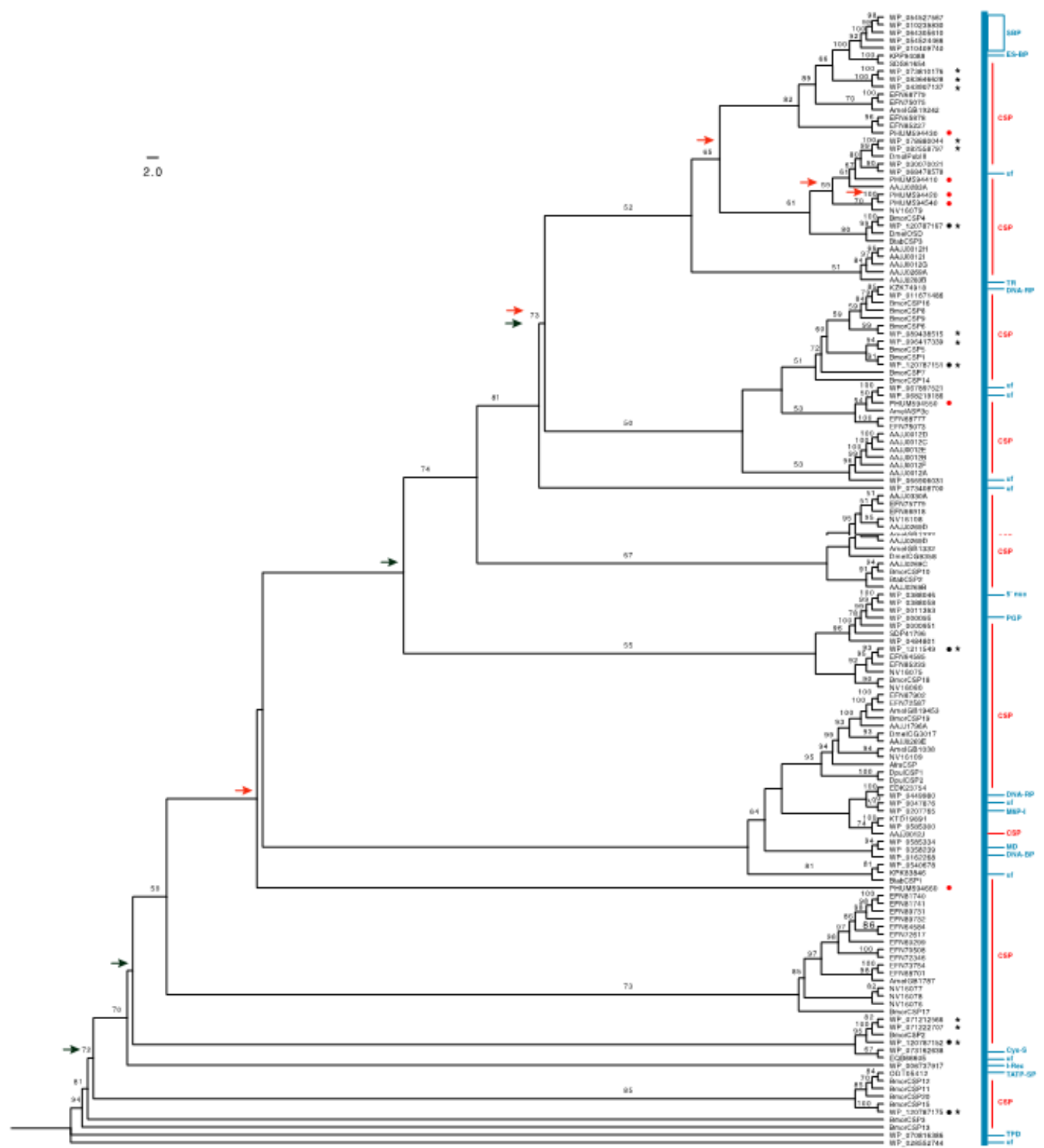

Figure 7: Phylogenetic analysis of CSPs and CSP-related gene families in bacteria. CSPs are those from figure 5. CSP-related genes in bacteria are from Liu and Picimbon [64]. Bootstrap values $>50 \%$ after 1000 replicates are shown in support of the branching in IQ-TREE. 5'nuc: 5' nuclease, I-Rec: Type I deoxyribonuclease (DNase I), Cys-S: Cysteine synthase, DNA-BP: DNA-binding protein, DNA-RP: DNA-regulatory protein (transcriptional regulator), ES-BP: Extracellular solute-binding protein, FAD-BP: Oxidoreductase FAD-binding protein, M6P-I: Mannose-6-phosphate isomerase, MD: Malonate decarboxylase, PGP: Phosphoglycolate phosphatase, SBP: Substrate-binding protein, TATP: Twin-arginine translocation pathway signal protein, TPD: Thiamine-phosphate diphosphorylase, TR: Transcriptional/cell division repressor, uf: unknown function. Red dots indicate the position of PhumCSPs. Black dots indicate the position of Macrococcus (staphylococcus) CSPs. Black arrows indicate inferred gene-duplication events in bacteria. Red arrows indicate inferred gene-duplication events in insects. The asterisks in black show the position of all B-CSPs reported in our study. TPD (Thiaminephosphate diphosphorylase) falls at the bottom of the tree, attracting BmorCSP3, BmorCSP13 and a group of BmorCSPs (BmorCSP11, BmorCSP12, BmorCSP15, BmorCSP20) with B-CSP WP_120787175 and twin-arginine translocation pathway signal protein (ODT05412) (85\%).

functions of CSPs may be linked to a transmembrane protein similar to a toll-like receptor such as WP_096417339 and later on evolved through new functions through loss or truncation of the transmembrane component. The tree is also showing a very high bootstrap value for soluble BmorCSP1 and WP_120787151
(91\%), and for soluble BmorCSP6 and WP_089438515 (99\%), strongly suggesting that the coming of soluble CSP proteins from membrane has happened not in Lepidoptera, Phthiraptera or Mallophaga, not in insects or some other arthropod species, but in the bacterial prokaryote cell. 
The CSP (OS-D or p10) multigene protein family is gigantic, perhaps an amazing summary of the cell or organism evolution. From their findings in Periplaneta regenerating legs (p10) and Drosophila olfactory sensilla (OS-D) to their high diversity in a bacterial strain such as A. baumannii or M. caseolyticus, several questions arise. How many organisms express them? Where are they found and in which conditions are they expressed? How could this protein cause a cell to respond to xenobiotic chemical, plant metabolite or infectious agent? And, most pressing of all, what are their functions? A gene family that is a group of genes that share important characteristics, similar sequences of DNA building blocks (nucleotides), providing instructions for making products (proteins) that have a similar structure, motif or function, not only in insects and arthropods, but also in microbes and bacteria, certainly retains crucial importance for functional evolution in cells eventually compatible with the beginning of life.

We do not know if insect is evolved from bacteria or if LUCA and all his descendants from birds to reptiles have CSPs. However, the common pattern of evolution in CSPs we report here in a comparative analysis of lice and some infectious bacterial agents is very intriguing in the fact that the protein structure is conserved from Acinetobacter to louse. Lice have been parasitizing human and mammals since Prehistory and the origin of human lice and their role in vector pathogenic bacteria that cause human infectious disease may date as far as $>6$ Mya [75]. Ape body or head, louse and the infectious bacterium they carry probably show an intrinsic association in a faithful long and complex evolutionary history that can be traced back to 20-25 Mya. Bacteria provide essential vitamins to the lice, and the lice pass on diseases to humans. Symbiotic bacterial evolution is driven by highly specific associations with the louse, and bacterium/louse evolution is tied to mammal/human host [76]. We speculate that CSPs had a key role to play in the evolutionary history of bacteria residing in lice and later in the infectious process of lice parasitizing the mammalian/human body or head.

Considering more the association of this group of genes with human diseases, a related point to consider is that the CSP structure triggers plant immune responses when injected into the phloem, similarly to honeydew-associated microbes [77,78,79]. While CSP and/or honeydew microbes help release phytoalexins, jasmonic acid, salicylic acid and volatile organic compounds (VOCs) from the leaves, it would be interesting to see if the injection of CSP structures and/or the inoculation of specific bacteria such as $A$. baumannii, M./S. caseolyticus, K. griseola and E. coli result in the activation of similar defense genes in the human tissue. The effects of CSPs in plants may be mediated by their ability to transport lipid and benzoic types of compounds as shown in whiteflies [28]. Lipid acid and benzoic acid are precursors of plant hormones in the jasmonate and salycilate class, respectively. Therefore, it is likely that CSPs can help stimulate specific hormone pathways by stimulating the transport of small precursor molecules. Whether this could be beneficial to treat some diseases related to lipid metabolism in human needs to be investigated further about the effects of injection of insect/bacterial CSPs through or via the human or mammalian skin. Lipid transporters are found in almost every type of intracellular organelle, but individual transporters have multiple functions, which strongly depend on the cell or tissue function [80]. It could be that a treatment with CSPs helps transport of lipids in the blood, lymph and body fluids, facilitating the dispersal of lipid droplets and chylomicrons after digestion. Combining CSPs to natural bacterial bio-products that target the gut flora may be an extremely beneficial approach for the treatment of obesity and other metabolic diseases [81]. There are numerous indications of an active lipid metabolism that is set out between bacteria and human cells or tissues. It has been proposed that this hijacking of lipids by the bacteria may be a way for the infectious agent to degrade host lipids while enriching its own stocks to survive, adapt and propagate to various tissues developing even more severe infection [82]. Thus, inhibiting B-CSPs may stop bacteria from hijacking human lipids. Then, an agent or a molecule capable to block B-CSPs in a specific manner may be able to influence the speed of recovery from an infectious disease. Lipid biological functions in bacteria are fuel for the regulation of cellular function, but mainly concern the membrane cell processes, i.e. selective permeability for exchange of essential water-soluble nutrients with the environment, cell division and cell protection [83]. So, altering the B-CSPs may interfere with the lipid pathways in the bacterial cell, affecting not only the membrane functions but also the cellular processes, which could be useful to counteract, exhaust or kill the defence mechanisms of particularly harmful microbial strains.

In addition, investigations about the function and binding properties of B-CSPs may open other new fields of research in medical microbiology. We may be able to increase the major beneficial activities of bacteria by cloning a specific CSP or CSP variant in a selection of microorganisms particularly useful or effective for human health protection $[12,13,14,81,82,83]$. We may also be able to develop new methods to knockdown specific CSP genes in toxic bacterial microorganisms, resulting not only in the alteration of lipid transport in the target bacterial cell, but also in the reduction of antibiotics/bactericide/germicide resistance and virulence in drug resistant strains. This area of research may bring some important concepts in cancer cell drug resistance [84]. It may also help understand the links between lipid metabolism and insulin resistance [85]. Therefore, controlling the function of bacterial CSPs appears to be in many ways a new very promising approach for controlling the pathogenicity of harmful bacteria such as A. baumannii, E. coli, Staphylococcus and Streptococcus, among many others, as well as for seeing the interplay between lipids and drugs. A modern strategy of louse pest control may also consist in altering the physiology of the symbiotic bacterial cell, disrupting the main vitamin or fuel supply of the parasite. Our finding of the existence of the protein gene family, CSPs, in so numerous bacteria genera that belong to infectious pathogens and/or insect-bacteria symbiosis is crucial to treat human diseases by two means, i.e. targeting a component of bacterial cells and/or eliminating the infectious agent necessary for the growth of many various human body parasites. 


\section{Acknowledgements}

Natural Sciences Foundation of Shandong Province (ZR2011CM046) and Shandong Province Overseas High-Level Talents Program (Taishan scholar, No. tshw20091015)

\section{References}

1. Lamarck JB. Philosophie Zoologique, Germer Bailliere. Paris. 1830.

2. Darwin C. On the Origin of Species by Means of Natural Selection, or the Preservation of Favoured Races in the Struggle for Life, Nature. 1859;5:502.

3. Owen R. Review of Darwin's Origin of Species. Edinburg Rev. 1860;3:487-532.

4. Vogt RG, Riddiford LM. Pheromone binding and inactivation by moth antennae. Nature. 1981;293:161-163.

5. Picimbon JF, Leal WS. Olfactory soluble proteins of cockroaches. Insect Biochem Mol Biol. 1999;29(11):973-978.

6. Angeli S, Ceron F, Scaloni A, Monti M, Monteforti G, Minnocci $A$, et al. Purification, structural characterization, cloning and immunocy tochemical localization of chemoreception proteins from Schistocerca gregaria. Eur J Biochem. 1999;262(3):745-754.

7. Picimbon JF. Biochemistry and evolution of CSP and OBP proteins. In: Blomquist GJ, Vogt RG, editors. Insect Pheromone Biochemistry and Molecular Biology, the Biosynthesis and Detection of Pheromones and Plant Volatiles. Elsevier Academic Press, London, San Diego. 2003;539-566.

8. Lartigue A, Campanacci V, Roussel A, Larsson AM, Jones TA, Tegoni M, et al. X-ray structure and ligand binding study of a moth chemosensory protein. J Biol Chem. 2002;277:32094-32098.

9. Jansen S, Zídek L, Löfstedt C, Picimbon JF, Sklenar V. 1H, 13C, and $15 \mathrm{~N}$ resonance assignment of Bombyx mori chemosensory protein 1 (BmorCSP1). J Biomol NMR. 2006;36:47.

10. Jansen S, Chmelik J, Zídek L, Padrta P, Novak P, Zdrahal Z, et al. Structure of Bombyx mori Chemosensory Protein 1 in solution. Arch Insect Biochem Physiol. 2007;66(3):135-145.

11. Tomaselli S, Crescenzi O, Sanfelice D, Ab E, Wechselberger R, Angeli $\mathrm{S}$, et al. Solution structure of a chemosensory protein from the desert locust Schistocerca gregaria. Biochemistry. 2006;45:16061613.

12.Xuan N, Bu X, Liu YY, Yang X, Liu GX, Fan ZX, et al. Molecular evidence of RNA editing in Bombyx chemosensory protein family. PLoS ONE. 2014; 9(2):e86932.

13. Xuan N, Rajashekar B, Kasvandik S, Picimbon JF. Structural components of chemosensory protein mutations in the silkworm moth, Bombyx mori. Agri Gene. 2016;2:53-58.

14.Xuan N, Rajashekar B, Picimbon JF. DNA and RNA-dependent polymerization in editing of Bombyx chemosensory protein (CSP) gene family. Agri Gene. 2019;12:100087.

15. Picimbon JF. Mutations in the insect transcriptome. J Clin Exp Pathol. 2016;6:3.

16. Picimbon JF. A new view of genetic mutations. Australas Med J. 2017;10:701-715.
17. Picimbon JF. Evolution of protein physical structures in insect chemosensory systems. In: Picimbon JF, editor. Olfactory Concepts of Insect Control-Alternative to Insecticides (volume 2). Springer Nature Switzerland. 2019; 231-263.

18.Campanacci V, Lartigue A, Hällberg BM, Jones TA, Giuici-Orticoni MT, Tegoni M, et al. Moth chemosensory protein exhibits drastic conformational changes and cooperativity on ligand binding. Proc Natl Acad Sci USA. 2003;100(9):5069-5074.

19. Nomura A, Kawasaki K, Kubo T, Natori S. Purification and localization of $\mathrm{p} 10$, a novel protein that increases in nymphal regenerating legs of Periplaneta americana (American cockroach). Int J Dev Biol. 1992;36(3):391-398.

20. Picimbon JF, Dietrich K, Breer H, Krieger J. Chemosensory proteins of Locusta migratoria (Orthoptera :Acrididae). Insect Biochem Mol Biol. 2000;30(3):233-241

21. Picimbon JF, Dietrich K, Angeli S, Scaloni A, Krieger J, Breer H, et al. Purification and molecular cloning of chemosensory proteins from Bombyx mori. Arch Insect Biochem Physiol. 2000;44(3):120-129.

22. Celorio-Mancera MdP, Sundmalm SM, Vogel H, Rutishauser D, Ytterberg AJ, Zubarv RA, et al. Chemosensory proteins, major salivary factors in caterpillar mandibular glands. Insect Biochem Mol Biol. 2012;42:796-805.

23. González-Caballero N, Valenzuela JG, Ribeiro JMC, Cuervo P, Brazil RP. Transcriptome exploration of the sex pheromone gland of Lutzomyia longipalpis (Diptera: Psychodidae: Phlebotominae). Parasit Vect. 2013;6:56.

24. Liu YL, Guo H, Huang LQ Pelosi P, Wang CZ. Unique function of a chemosensory protein in the proboscis of two Helicoverpa species. J Exp Biol. 2014;217:1821-1826.

25. Perkin LC, Friesen KS, Flinn PW, Oppert B. Venom gland components of the ectoparasitoid wasp, Anisopteromalus calandrae. J Venom Res. 2015;6:19-37.

26.Zhu J, Iovinella I, Dani FR, Liu YL, Huang LQ, Liu Y, et al. Conserved chemosensory proteins in the proboscis and eyes of Lepidoptera. Int J Biol Sci. 2016;12:1394-1404.

27. Xuan N, Guo X, Xie HY, Lou QN, Bo LX, Liu GX, et al. Increased expression of CSP and CYP genes in adult silkworm females exposed to avermectins. Insect Sci. 2015;22(2):203-219.

28. Liu GX, Ma HM, Xie YN, Xuan N, Xia G, Fan ZX, et al. Biotype characterization, developmental profiling, insecticide response and binding property of Bemisia tabaci chemosensory proteins: role of CSP in insect defense. PLoS ONE. 2016; 11(5):e0154706.

29. Sabatier L, Jouanguy E, Dostert C, Zachary D, Dimarcq JL, Bulet P, et al. Pherokine-2 and -3: Two Drosophila molecules related to pheromone/ odor-binding proteins induced by viral and bacterial infections. Eur J Biol. 2003;270:3398-3407.

30. Picimbon JF, DietrichK, Krieger J, Breer H. Identity and expression pattern of chemosensory proteins in Heliothis virescens (Lepidoptera, Noctuidae). Insect Biochem Mol Biol. 2001;31(12):1173-1181. 
31. Wanner KW, Isman MB, Feng Q Plettner E, Theilmann DA. Developmental expression patterns of four chemosensory protein genes from the Eastern spruce budworm, Choristoneura fumiferana. Insect Mol Biol. 2005;14(3):289-300.

32. Maleszka J, Forêt S, Saint R, Maleszka R. RNAi-induced phenotypes suggest a novel role for a chemosensory protein CSP5 in the development of embryonic integument in the honeybee (Apis mellifera). Dev Genes Evol. 2007;217(3):189-196.

33. Guo W, Wang XH, Ma ZY, Xue L, Han JY, et al. CSP and Takeout genes modulate the switch between attraction and repulsion during behavioral phase change in the migratory locust. PLoS Genet. 2011;7:e1001291.

34. Liu GX, Ma HM, Xie HY, Xuan N, Picimbon JF. Sequence variation of Bemisia tabaci Chemosensory Protein 2 in cryptic species B and Q: new DNA markers for whitefly recognition. Gene. 2016;576:284-291.

35. Pedra JH, Brandt A, Li HM, Westerman R, Romero-Serverson J, Pollack $\mathrm{RJ}$, et al. Transcriptome identification of putative genes involved in protein catabolism and innate immune response in human body louse (Pediculicidae: Pediculus humanus). Insect Biochem Mol Biol. 2003;33(11):1135-1143.

36. Kirkness EF, Haas BJ, Sun W, Braig HR, Perotti MA, Clark JM, et al. Genome sequences of the human body louse and its primary endosymbiont provide insights into the permanent parasitic lifestyle. Proc Natl Acad Sci USA. 2010;107(27):12168-12173.

37. Wanner KW, Willis LG, Theilmann DA, Isman MB, Feng Q Plettner E. Analysis of the insect os-d-like gene family. J Chem Ecol. 2004;30(5):889-911.

38. Honeybee Genome Sequencing Consortium. Insights into social insects from the genome of the honeybee Apis mellifera. Nature 2006;443:931-949.

39. Forêt S, Wanner K, Maleszka R. Chemosensory proteins in the honeybee: insights from the annotated genome, comparative analysis and expressional profiling. Insect Biochem Mol Biol. 2007;37(1):1928.

40. Bonasio R, Zhang G, Ye C, Mutti NS, Fang X, Qin N, et al. Genomic comparison of the ants Camponotus floridanus and Harpegnathos saltator. Science. 2010;329:1068-1071.

41. Kulmuni J, Wurm Y, Pamilo P. Comparative genomics of chemosensory protein genes reveals rapid evolution and positive selection in antspecific duplicates. Heredity. 2013;110(6):538-547.

42. Zhao Y, Wang F, Zhang X, Zhang S, Guo S, Zhu G, et al. Transcriptome and expression patterns of chemosensory genes in antennae of the parasitoid wasp Chouioia cunea. PLoS ONE. 2016;11:e0148159.

43. Liu GX, Arnaud P, Offmann B, Picimbon JF. Genotyping and bio-sensing chemosensory proteins in insects. Sensors. 2017;17(8): 1801.

44. Liu GX, Xuan N, Rajashekar B, Arnaud P, Offmann B, Picimbon JF. Genetic plasticity of insect chemosensory proteins. Front Genet. submitted.

45. Edgar RC. MUSCLE: multiple sequence alignment with high accuracy and high throughput. Nucleic Acids Res. 2004;32(5):1792-1797.

46. Nguyen LT, Schmidt HA, von Haeseler A, Minh BQ. IQ-TREE: a fast and effective stochastic algorithm for estimating maximum-likelihood phylogenies. Mol Biol Evol. 2015;32(1):268-274.

47. Fig Tree, a graphical viewer of phylogenetic trees.

48. Wong TY, Fernandes S, Sankhon N, Leong PP, Kuo J, Liu JK. Role of premature stop codons in bacterial evolution. J Bacteriol. 2008;190:6718-6725.

49. Fiszer D, Rozwadowska N, Rychlewski L, Kosicki W, Kurpisz M. Identification of IL-18RAP mRNA truncated splice variants in human testis and the other human tissues. Cytokine. 2007;39(3):178-183.

50. Yun JH, Roh SW, Whon TW, Jung MJ, Kim MS, Park DS, et al. Insect gut bacterial diversity determined by environmental habitat, diet, developmental stage, and phylogeny of host. Appl Environ Microbiol. 2014;80:5254-5264.

51. Book AJ, Lewin GR, McDonald BR, Takasuka TE, Doering DT, Adams AS, et al. Cellulolytic Streptomyces strains associated with herbivorous insects share a phylogenetically linked capacity to degrade lignocellulose. Appl Environ Microbiol. 2014;80:4692-4701.

52. Gonzales-Escobar JL, Grajales-Lagunes A, Smolinski A, Chagolla-Lopez A, De Leon-Rodriguez A, Barba de la Rosa AP. Microbiota of edible Liometopum apiculatum ant larvae reveals potential functions related to their nutritional value. Food Res Int. 2018;109:497-505.

53. Salzman S, Whitaker M, Pierce NE. Cycad-feeding insects share a core gut microbiome. Biol J Linn Soc. 2018;123:728-738.

54. Paniagua Voirol LR, Frago E, Kaltenpoth M, Hilker M, Fatouros NE. Bacterial symbionts in Lepidoptera: their diversity, transmission, and impact on the host. Front Microbiol. 2018;9:556. doi: 10.3389/ fmicb.2018.00556

55. Engel P, Moran N. The gut microbiota of insects-diversity in structure and function. FEMS Microbiol Rev. 2013;37(5):699-735.

56. La Scola B, Raoult D. Acinetobacter baumannii in human body louse. Emerg Infect Dis. 2004;10:1671-1673.

57. Sunantaraporn S, Sanprasert V, Pengsakul T, Phumee A, Boonserm R, Tawatsin A, et al. Molecular survey of the head louse Pediculus humanis capitis in Thailand and its potential role for transmitting Acinetobacter spp. Parasit Vect. 2015;8:127.

58. Araujo A, Ferreira LF, Guidon N, Maues Da Serra Freire N, Reinhard KJ, Dittmar K. Ten thousand years of head lice infection. Parasitol Today. 2000;16(7):269.

59. Badiaga S, Brouqui P. Human louse-transmitted infectious diseases. Clin Microbiol Infect. 2012;18(4):332-337.

60. Koonin EV. The origin of introns and their role in eukaryogenesis: a compromise solution to the introns-early versus intron late debate? Biol Direct. 2006;1:22.

61. Cooper GM. The origin and evolution of cells. In: The cell: a molecular approach, 2nd edition, Sunderland (MA), Sinauer Associates, 2000;689.

62. Briand L, Swasdipan N, Nespoulos C, Bezirard V, Blon F, Huet JC, et al. Characterization of a chemosensory protein (ASP3c) from honeybee (Apis mellifera L.) as a brood pheromone carrier. Eur J Biochem. 2002;269:4586-4596.

63. Sprusansky 0, Zhou L, Jordan S, White J, Westpheling J. Identification of three new genes involved in morphogenesis and antibiotic production in Streptomyces coleicolor. J Bacteriol. 2003;185(20):6147-6157. doi: 10.1128/JB.185.20.6147-6157.2003 
64. Vollmer J, Schiefer A, Schneider T, Julicher K, Johnston KL, Taylor MJ, et al. Requirement of lipid II biosynthesis for cell division in cell wall-less Wolbachia, endobacteria of arthropods and filial nematodes. Int J Med Microbiol. 2013;303:140-149. doi: 10.1016/j.ijmm.2013.01.002

65. Wybouw N, Dermauw W, Tirry L, Stevens C, Grbic M, Feyereisen R, et al. A gene horizontally transferred from bacteria protects arthropods from host plant cyanide poisoning. Elife. 2014;3:e02365. doi: 10.7554/eLife.02365

66. Downs AMR, Stafford KA, Coles GC, Downs AMR, Stafford KA, Coles GC. Head lice: prevalence in schoolchildren and insecticide resistance. Trends Parasitol. 1999;15(1):P1-4.

67. Downs AMR, Stafford KA, Hunt LP, Ravenscroft JC, Coles GC. Widespread insecticide resistance in head lice to the over-the-counter pediculocides in England, and the emergence of carbaryl resistance. B J Dermatol. 2002;146:88-93.

68. Thomas DRh, McCarroll L, Roberts R, Karunaratne P, Roberts C, Casey D, et al. Surveillance of insecticide resistance in headlice using biochemical and molecular methods. Arch Dis Child. 2006;91:777778. doi: 10.1136/adc.2005.091280

69. Durand R, Bouvresse S, Berdjane Z, Izri A, Chosidow O, Clark JM. Insecticide resistance in head lice: clinical, parasitological and genetic aspects. Clin Microbiol Infect. 2012;18(4):338-344. doi: 10.1111/j.1469-0691.2012.03806.x

70. Drali R, Benkouiten S, Badiaga S, Bitam I, Rolain JM, Brouqui P. Detection of a knockdown resistance mutation associated with permethrin resistance in the body louse Pediculus humanis corporis by use of melting curve analysis genotyping. J Clin Microbiol. 2012;50(7):22292233. doi: 10.1128/JCM.00808-12

71. Cloete TE. Resistance mechanism of bacteria to antimicrobial compounds. Int Biodeter Biodegr. 2003;51(4):277-282.

72. Wyrsch ER, Chowdhury R, Chapman TA, Charles IG, Hammond JM, Djordjevic SP. Genomic microbial epidemiology is needed to comprehend the global problem of antibiotic resistance and to improve pathogen diagnosis. Front Microbiol. 2016:7:843. doi: 10.3389/fmicb.2016.00843

73. Yurtsev EA, Conwill A, Gore J. Oscillatory dynamics in a bacterial crossprotection mutualism. Proc Natl Acad Sci USA. 2016;113(22):62366241. doi: $10.1073 /$ pnas. 1523317113
74. Liu GX, Picimbon JF. Bacterial origin of insect chemosensory odorbinding proteins. Gene Transl Bioinform. 2017; 3:e1548.

75. Boutellis A, Abi-Rached L, Raoult D. The origin and distribution of human lice in the world. Infect Genet Evol. 2014;23:209-217. doi: 10.1016/j.meegid.2014.01.017

76. Boyd BM, Allen JM, Nguyen NP, Vachaspati P, Quickcall Z, Warnow T, et al. Primates, lice and bacteria: speciation and genome evolution in the symbionts of hominid lice. Mol Biol Evol. 2017;34(7):1743-1757. doi: 10.1093/molbev/msx117

77. Bos JI, Prince D, Pitino M, Maffei ME, Win J, Hogenhout SA. A functional genomics approach identified candidate effectors from the aphid species Myzus persicae (green peach aphid). PLoS Genet. 2010;6(11):e1001216. doi: 10.1371/journal.pgen.1001216

78. Rodriguez PA, Stam R, Warbroek T, Bos JI. Mp10 and Mp42 from the aphid species Myzus persicae trigger plant defenses in Nicotiana benthemiana through different activities. Mol Plant Microbe Interact. 2014;27(1):30-39. doi: 10.1094/MPMI-05-13-0156-R

79. Wari D, Kabir MA, Mujiono K, Hojo Y, Shinya T, Tani A, et al. Honeydewassociated microbes elicit defense responses against brown planthopper in rice. J Exp Bot. 2019;70(5):1683-1696. doi: 10.1093/ jxb/erz041

80. Tarling EJ, de Aguiar Vallim TQ Edwards PA. Role of ABC transporters in lipid transport and human disease. Trends Endocrinol Metabol. 2013; 24: 342-350.

81. Yue S, Chinnapandi B, Ge H, Zou X, Chen X, Wang C, et al. A lactobacillus cocktail changes gut flora and reduces cholesterolemia and weight gain in hyperlipidemia mice. SOJ Microbiol Infect Dis. 2014;2(2):1-14.

82. Gajenthra Kumar N, Contaifer D Jr, Baker PRS, Ekroos K, Jefferson KK, Wijesinghe DS. Untargeted lipidomic analysis to broadly characterize the effects of pathogenic and non-pathogenic staphylococci on mammalian lipids. PLoS ONE. 2018;13(10):e0206606.

83. Barak I, Muchova K. The role of lipid domains in bacterial cell processes. Int J Mol Sci. 2013;14(2):4050-4065. doi: 10.3390/ijms14024050

84. Iwamoto H, Abe M, Yang Y, Cui D, Seki T, Nakamura M, et al. Cancer lipid metabolism confers antiangiogenic drug resistance. Cell Metabol. 2018;28(1):104-117.e5. doi: 10.1016/j.cmet.2018.05.005

85. Savage DB, Petersen KF, Shulman GI. Disordered lipid metabolism and the pathogenesis of insulin resistance. Physiol Rev. 2007;87(2):507520 . 